\title{
Feedforward Inhibition Underlies the Propagation of Cholinergically Induced Gamma Oscillations from Hippocampal CA3 to CA1
}

\author{
Rita Zemankovics, ${ }^{1}$ Judit M. Veres, ${ }^{1}$ Iris Oren, ${ }^{2}$ and Norbert Hájos ${ }^{1}$ \\ ${ }^{1}$ Institute of Experimental Medicine, Hungarian Academy of Sciences, H-1450, Budapest, Hungary, and ${ }^{2}$ Centre for Cognitive and Neural Systems, \\ University of Edinburgh, Edinburgh EH8 9JZ, United Kingdom
}

Gamma frequency $(30-80 \mathrm{~Hz})$ oscillations are implicated in memory processing. Such rhythmic activity can be generated intrinsically in the $\mathrm{CA} 3$ region of the hippocampus from where it can propagate to the CA1 area. To uncover the synaptic mechanisms underlying the intrahippocampal spread of gamma oscillations, we recorded local field potentials, as well as action potentials and synaptic currents in anatomically identified CA1 and CA3 neurons during carbachol-induced gamma oscillations in mouse hippocampal slices. The firing of the vast majority of $\mathrm{CA} 1$ neurons and all $\mathrm{CA} 3$ neurons was phase-coupled to the oscillations recorded in the stratum pyramidale of the CA1 region. The predominant synaptic input to CA1 interneurons was excitatory, and their discharge followed the firing of CA3 pyramidal cells at a latency indicative of monosynaptic connections. Correlation analysis of the input-output characteristics of the neurons and local pharmacological block of inhibition both agree with a model in which glutamatergic CA3 input controls the firing of CA1 interneurons, with local pyramidal cell activity having a minimal role. The firing of phase-coupled CA1 pyramidal cells was controlled principally by their inhibitory inputs, which dominated over excitation. Our results indicate that the synchronous firing of CA3 pyramidal cells rhythmically recruits CA1 interneurons and that this feedforward inhibition generates the oscillatory activity in CA1. These findings identify distinct synaptic mechanisms underlying the generation of gamma frequency oscillations in neighboring hippocampal subregions.

\section{Introduction}

The temporal structure of neuronal firing has been implicated in information processing. Such precisely timed firing can generate oscillatory activities at different frequencies in the local field potential (LFP) (Paulsen and Moser, 1998). In cortical networks, including the hippocampus, gamma $(30-80 \mathrm{~Hz})$ oscillations have received particular attention because they are associated with sensory encoding, memory storage and retrieval, and attentive behavior (Singer, 1993; Montgomery and Buzsáki, 2007).

Gamma oscillations can be generated intrinsically in a neuronal circuit (Bragin et al., 1995; Csicsvári et al., 2003), or they can be evoked by extrinsic inputs (Bragin et al., 1995; Colgin et al., 2009; Minlebaev et al., 2011). One of the best examples for the

Received Aug. 1, 2012; revised May 27, 2013; accepted June 18, 2013.

Author contributions: R.Z. and N.H. designed research; R.Z. and J.M.V. performed research; R.Z., J.M.V., I.O., and N.H. analyzed data; R.Z. and N.H. wrote the paper.

This work was supported by the Wellcome Trust International Senior Research Fellowship, the Wellcome Trust, the National Office for Research and Technology (Grant OMFB-01678/2009), and the Hungarian Scientific Research Fund (Grant T49517). We are grateful to Prof. Ole Paulsen for helpful discussions and to Prof. Dimitri Kullmann and Dr. Mirko Santello for useful comments on this manuscript. We acknowledge Prof. Hanna Monyer, Dr. Gábor Szabó, and Ferenc Erdélyi for generously providing the transgenic mice. We thank Erzsébet Gregori for excellent technical assistance.

The authors declare no competing financial interests.

Correspondence should be addressed to Norbert Hájos, Institute of Experimental Medicine, Hungarian Academy

of Sciences, Budapest H-1450, Hungary. E-mail: hajos@koki.hu.

R. Zemankovics's present address: Department of Physiology, University of Bern, Bern, Switzerland.

DOI:10.1523/JNEUROSCI.3680-12.2013

Copyright $\odot 2013$ the authors $\quad 0270-6474 / 13 / 3312337-15 \$ 15.00 / 0$ latter case is the gamma oscillation in the CA1 region of the hippocampus, where these oscillations can be driven either by the afferents from the CA3 region or by the inputs from the entorhinal cortex (Bragin et al., 1995; Colgin et al., 2009). These two types of gamma oscillations in CA1, having different frequency characteristics, mutually exclude each other during theta rhythm (Colgin et al., 2009). Although the mechanisms underlying the intrinsically generated gamma oscillations within the hippocampal CA3 network have been elucidated (for review, see Hájos and Paulsen, 2009), the cellular and network mechanisms underlying their propagation to the downstream regions have not yet been investigated.

Carbachol (CCh), a cholinergic receptor agonist, can induce synchronous, gamma frequency activity in hippocampal slices, which shares many features with hippocampal gamma oscillations occurring in vivo (Fisahn et al., 1998; Csicsvári et al., 2003; Hájos and Paulsen, 2009). Studies of CCh-induced oscillations in CA3 have revealed that these oscillations are generated by a synaptic feedback loop comprising CA3 pyramidal cells and fast spiking basket cells (Mann et al., 2005; Gulyás et al., 2010). During in vitro gamma oscillations, the discharge of principal cells is controlled by perisomatic inhibition, whereas the firing of GABAergic interneurons is driven by precisely timed excitatory input (Oren et al., 2006). The frequency and the magnitude of these oscillations are primarily determined by the decay kinetics and the amplitude of perisomatic inhibitory currents (Fisahn et al., 1998; Oren et al., 2010). In the CA1 region, where extrinsic 
inputs drive these synchronous network activities (Bragin et al., 1995; Colgin et al., 2009), much less is known about the properties of gamma oscillations.

The goal of this study was to identify how gamma oscillations, generated intrinsically in CA3, spread to the CA1 area. To this end, we investigated the relationship between the firing activity and synaptic inputs of different cell types during CCh-induced network oscillations in hippocampal slices, combined with local drug application. We demonstrate that neuronal input-output relationships are consistent with a feedforward inhibitionmediated propagation of gamma frequency oscillations from the CA3 to the CA1 region.

\section{Materials and Methods}

Animals were kept and used according to the regulations of the European Community's Council Directive of November 24, 1986 (86/609/EEC), and experimental procedures were reviewed and approved by the Animal Welfare Committee of the Institute of Experimental Medicine, Hungarian Academy of Sciences, Budapest, Hungary.

CD1 mice of both sexes (postnatal days 15-23) were used in most of the experiments. To measure selectively from cells containing the $\mathrm{Ca}^{2+}$ binding protein parvalbumin (PV), transgenic mice on an FVB/ $\mathrm{N}$ background expressing the enhanced green fluorescent protein (eGFP) controlled by PV promoter (Meyer et al., 2002) were also used (postnatal days 15-21). Mice were decapitated under deep isoflurane anesthesia. The brain was removed into an ice-cold cutting solution, which had been bubbled with $95 \% \mathrm{O}_{2} / 5 \% \mathrm{CO}_{2}$ (carbogen gas) for at least $30 \mathrm{~min}$ before use. The cutting solution contained (in $\mathrm{mm}$ ) 205 sucrose, $2.5 \mathrm{KCl}, 26$ $\mathrm{NaHCO}_{3}, 0.5 \mathrm{CaCl}_{2}, 5 \mathrm{MgCl}_{2}, 1.25 \mathrm{NaH}_{2} \mathrm{PO}_{4}$, and 10 glucose, saturated with $95 \% \mathrm{O}_{2} / 5 \% \mathrm{CO}_{2}$. Horizontal hippocampal slices of $450 \mu \mathrm{m}$ thickness were cut using a vibratome (VT1000S; Leica). Care was taken to remove the entorhinal cortical regions from the slices.

After acute slice preparation, the slices were placed into an interfacetype holding chamber for recovery. This chamber contained standard $\mathrm{aCSF}$ at $35^{\circ} \mathrm{C}$ that gradually cooled down to room temperature. The aCSF had the following composition (in mM): $126 \mathrm{NaCl}, 2.5 \mathrm{KCl}, 26 \mathrm{NaHCO}_{3}$, $2 \mathrm{CaCl}_{2}, 2 \mathrm{MgCl}_{2}, 1.25 \mathrm{NaH}_{2} \mathrm{PO}_{4}$, and 10 glucose, saturated with $95 \%$ $\mathrm{O}_{2} / 5 \% \mathrm{CO}_{2}$. After incubation for a minimum of $1 \mathrm{~h}$, slices were transferred individually to a submerged-style recording chamber. We used a modified custom-made recording chamber with a dual superfusion system for improved metabolic supply to the slices (Hájos et al., 2009). In this design, the slices were placed on a mesh, and two separate fluid inlets allowed aCSF to flow both above and below the slices with a rate of 3-3.5 $\mathrm{ml} / \mathrm{min}$ for each flow channel at $30-32^{\circ} \mathrm{C}$.

Standard patch electrodes were used in all recording configurations (i.e., whole-cell patch-clamp, loose-patch, and field potential recordings). Pipette resistances were 3-6 M $\Omega$ when filled either with the intrapipette solution or with aCSF. The intrapipette solution contained (in $\mathrm{mm}) 138 \mathrm{~K}$-gluconate, $3 \mathrm{CsCl}, 10$ disodium creatine phosphate, $4 \mathrm{Mg}$ ATP, 0.4 Tris-GTP, 10 HEPES, and 0.2 QX 314 (pH 7.38; 285 $\left.\mathrm{mOsm} \cdot 1^{-1}\right)$. For later morphological identification of the recorded cells, biocytin in a concentration of $3-5 \mathrm{mg} / \mathrm{ml}$ was added to the pipette solution freshly before use.

Data acquisition. Data were recorded with a Multiclamp 700B amplifier (Molecular Devices). As a first step, two pipettes filled with aCSF were placed into the hippocampal slice preparation: one into the stratum pyramidale of the CA1 area and another into the stratum pyramidale of the CA3b area. After $\sim 10-15$ min of bath-applied 5-10 $\mu \mathrm{M} \mathrm{CCh,} \mathrm{which} \mathrm{was}$ usually enough time to induce stable, persistent oscillations in the slices (Hájos and Mody, 2009), the field potentials were recorded simultaneously on two channels for at least $120 \mathrm{~s}$, with the aim to compare the local field potential oscillations between the two regions. Then the electrode was removed from the CA3 area, while the electrode in CA1 was left in the same position. As a next step, in addition to the recording of local field potential in CA1, action potentials were detected extracellularly from individual neurons of CA1 or CA3 with the second pipette filled with aCSF. The loose-patch recordings were visually guided using differential interference contrast microscopy (BX61W; Olympus), and action poten- tials were detected for $60-120 \mathrm{~s}$, depending on the firing frequency of the cell. This pipette was then withdrawn from the slice, and whole-cell patch-clamp recordings were performed on the same cells with a new pipette filled with a $\mathrm{K}^{+}$-based intrapipette solution.

In the experiments involving local block of inhibition, the oscillation was induced as described above. A third pipette filled with a $G_{A B A}$ receptor antagonist, SR-95531 (gabazine, $50 \mu \mathrm{M}$ ), was placed above the stratum pyramidale in CA1. To reduce the spread of gabazine into the CA3 region, the slices were positioned in the recording chamber such that aCSF flowed from CA3 to CA1. After inducing and recording stable oscillations in both CA3 and CA1, the CA1 pipette was moved to record the firing activity of a CA1 cell in a loose-patch mode. After recording the baseline firing activity of the CA1 neuron for 60-120 s, a 2-min-long gabazine puff was applied. In the case of PV + interneurons after a few minutes of recovery time, the recording pipette filled with aCSF was withdrawn and replaced by a new patch pipette filled with a $\mathrm{K}^{+}$-based intrapipette solution to record synaptic currents in the same cell in a whole-cell voltage-clamp configuration both under control conditions (i.e., in the presence of 5-10 $\mu \mathrm{M} \mathrm{CCh}$ ) and during local gabazine application.

Access resistance was in the range of 5-20 M $\Omega$ and was compensated (65-75\%). Only recordings where the access resistance did not change substantially $(>25 \%)$ were included in the study. Reported values of voltage measurements were not corrected for the junction potential. To record EPSCs and IPSCs, cells were voltage clamped at a holding potential of the estimated reversal potential for IPSCs (approximately -70 $\mathrm{mV})$ and EPSCs $(\sim 0 \mathrm{mV})$, respectively. Both field and unit recordings were low-pass filtered at $2 \mathrm{kHz}$ using the built-in Bessel filter of the amplifier. Data were digitized at $6 \mathrm{kHz}$ with a PCI-6042E board (National Instruments) and EVAN 1.3 software and analyzed off-line with Igor Pro 5.01 software (Wavemetrics) using either standard or custom-made Igor Pro procedures.

Event detection and analysis. Recordings were further filtered off-line using a digital, bidirectional, phase-conserving filter. Field recordings were low-pass filtered at $1 \mathrm{kHz}$, extracellular unit recordings were highpass filtered at $40 \mathrm{~Hz}$ to isolate spikes, and whole-cell recordings of postsynaptic currents (PSCs) were high-pass filtered at $1 \mathrm{~Hz}$ to filter out slow fluctuations in the holding current.

The power of the field oscillation was calculated with power spectral density (PSD) analysis of $\sim 60$-s-long field recordings. Before the fast Fourier transform was performed, time windows of $\sim 1.5 \mathrm{~s}$ with $50 \%$ overlap were multiplied by a Hanning window to minimize the end effects. The area under the power spectral density curve between 15 and $45 \mathrm{~Hz}$ was taken as the power of the gamma frequency oscillation.

To extract the magnitude and the phase of the different frequency components of the field oscillation and to get information on changes in frequency and amplitude of the periodic signal with time, wavelet analysis using a Morlet-wavelet basis was used. The wavelet transform of the field recording was examined between 10 and $45 \mathrm{~Hz}$ with scales chosen to reflect the equivalent Fourier frequency (Le Van Quyen et al., 2001). For each time point, the maximum of the wavelet transform magnitude was found, and the corresponding dominant frequency was identified. The phase of the time point was defined in terms of the dominant frequency. Phase was defined in radians such that $-\pi$ was associated with the minimum of the oscillation, and a full cycle ran from $-\pi$ to $\pi$. Cells for which the wavelet magnitude of the field oscillation changed by $>2$ SDs between spike train and PSC recordings were excluded from the study.

Event times for action potentials were defined as the time of crossing a voltage threshold set by visual inspection to exceed the noise level. The mean firing rate for cells was calculated as the total number of events during the recording epoch divided by the length of the epoch. Normalized spiking frequency was calculated by dividing the mean firing rate by the frequency of the oscillation. Event phases were defined as the wavelet phase of the dominant frequency at the time of the event. To calculate the probability of discharge of a given cell group, the event number versus phase histograms of each cell in the group were normalized by the maximal spike count, summed, and divided by the number of cells in the given cell group. This averaged phase histogram was then multiplied by the mean of the normalized spiking frequency for the given cell group. 
A
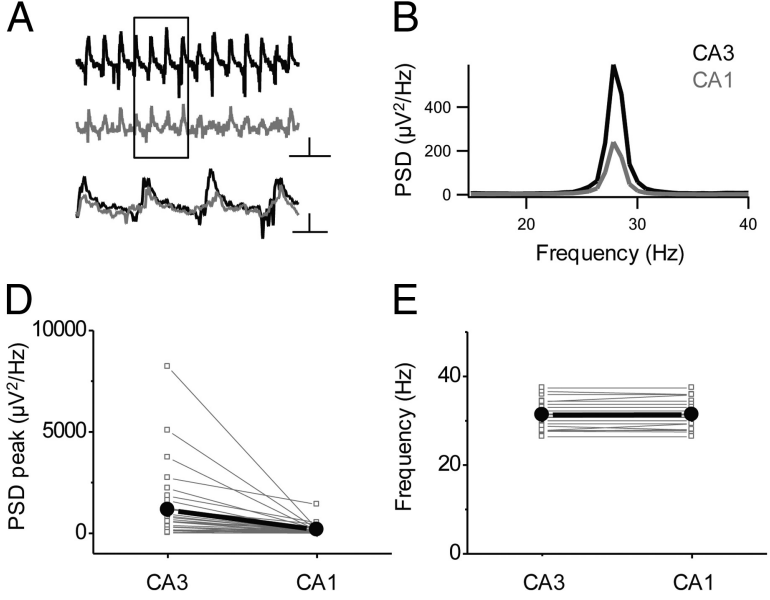

E
C

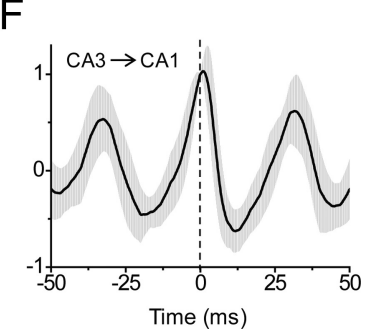

Figure 1. Comparison of field potential oscillations recorded extracellularly from the stratum pyramidale of the CA3 and CA1 regions of the hippocampus after bath application of $10 \mu \mathrm{m}$ carbachol. $A$, Raw traces recorded simultaneously from the stratum pyramidale in CA3 (top trace, black) and CA1 (middle trace, gray) after bath application of $\mathrm{CCh}$. The bottom trace is an expansion of the boxed area of the top traces showing the LFP recordings from CA3 (black) and CA1 (gray) overlapped. Calibration: vertical, 0.1 $\mathrm{mV}$; horizontal, $0.1 \mathrm{~ms}$ for upper traces and $0.02 \mathrm{~ms}$ for lower trace. $\boldsymbol{B}$, Power spectral density function of the traces in $\boldsymbol{A}$ showing a peak in the gamma frequency band. C, Cycle average of the field potential oscillation shown in $A . D, E$, Comparison of the power $(\boldsymbol{D})$ and frequency $(\boldsymbol{E})$ of oscillations in CA3 and CA1. Means are indicated with black dots. $\boldsymbol{F}$, Mean cross-correlation based on $60 \mathrm{~s}$ samples of CA3-CA1 LFP recordings. The shaded region around the mean represents \pm SD.

Event times for PSCs were defined as time of peak current per cycle of the oscillation, and these were converted to wavelet phases of the dominant frequency.

The cycle-averaged events (see Figs. 5, 6) (as well as the cycle-averaged field) were obtained by summing recordings over cycles, between $-\pi$ and $\pi$ and dividing by the number of cycles. Each cycle was linearly scaled to span $2 \pi$ radians regardless of the number of data points.

Phasic charge transfer was calculated in the following way: an initial baseline estimate was obtained by taking the maximum (for EPSCs) or minimum (for IPSCs) of the cycle-averaged events. Then for each $0.2 \mathrm{~s}$ epoch, the mean of all current values exceeding this initial baseline estimate was calculated. This mean was used as the baseline for the epoch. If no current value was found to exceed the initial baseline estimate during the epoch, the baseline estimate would increment negatively for EPSCs and positively for IPSCs until such current values were found. This calculation was repeated for all epochs of the entire recording. The integral from this baseline value was calculated over each cycle, and the mean of these integrals were taken as the phasic charge transfer.

The cycle-averaged PSCs were converted to excitatory $\left(g_{\mathrm{e}}\right)$ and inhibitory $\left(g_{\mathrm{i}}\right)$ conductances using the following equation:

$$
g_{e / i}=\frac{1}{\left(V_{h}-E_{e / i}^{r e v}\right)},
$$

where $I_{\mathrm{e} / \mathrm{i}}$ is the phasic excitatory/inhibitory current, $V_{\mathrm{h}}$ is the holding potential, and $E_{e / i}^{r e v}$ is the reversal potential for the conductance of interest. The conductances were used to estimate the net apparent reversal potential $\left(E_{s y n}^{r e v}\right)$ by solving the following equation:

$$
\begin{gathered}
\mathrm{I}_{\text {syn }}=\mathrm{g}_{\mathrm{e}}\left(\mathrm{E}_{\text {syn }}^{\mathrm{rev}}-\mathrm{E}_{\mathrm{e}}^{\mathrm{rev}}\right)+\mathrm{g}_{\mathrm{i}}\left(\mathrm{E}_{\text {syn }}^{\mathrm{rev}}-\mathrm{E}_{\mathrm{i}}^{\mathrm{rev}}\right)=0 \\
E_{\text {syn }}^{r e v}=\frac{g_{e} E_{e}^{r e v}+g_{i} E_{i}^{r e v}}{g_{e}+g_{i}} .
\end{gathered}
$$

In the experiments where gabazine was applied into CA1, the CA3 field recording was used as a reference for phase relationships of the events recorded in CA1 cells. Only recordings where the CA3 oscillation was stable (the wavelet magnitude of the field oscillation changed by $<2$ SDs during the entire course of the experiment) were included in the study.

Statistical analyses. The phase-coupling of the events was determined by using circular statistics. The strength of phase-coupling was calculated by summing all event phases within an epoch as unity vectors and dividing the resulting vector sum $(\vec{R})$ by the number of events (Zar, 1999). The length of this normalized vector $(r)$ was taken as the strength of the phasecoupling. If the phases of all unity vectors are identical, then $r$ is equal to 1 , whereas it is 0 in a case of uniform distribution. The mean event phase was defined as the direction of the resultant vector $(\phi)$.

The Rayleigh probability of $\vec{R}\left(p_{r}\right)$ was used to determine the significance of the phasecoupling. It was calculated by the following equation:

$$
\begin{aligned}
p_{r}= & e^{-z}\left(1+\frac{2 Z-Z^{2}}{4 n}\right. \\
& \left.-\frac{24 Z-132 Z^{2}+76 Z^{3}-9 Z^{4}}{288 n^{2}}\right),
\end{aligned}
$$

where $n$ is the number of spikes, and $Z=n r^{2}$ (Fisher, 1993). Events were considered to be phase-coupled if the Rayleigh test indicated that they were not distributed randomly around the gamma cycle $\left(p_{\mathrm{r}}<0.01\right)$ (Zar, 1999).

The circular SD was taken as follows:

$$
\sigma=\sqrt{-2 \ln r},
$$

where $r$ is the phase-coupling strength (Zar, 1999).

For linear data that were normally distributed according to the Kolmogorov-Smirnov test $(p>0.05)$, the equality of means of the measured variables of the different cell groups was tested by the Student's $t$ test or ANOVA. In the latter case, the Bonferroni's post hoc test was used to find significant differences between group means. For comparison of nonnormally distributed linear data ( $p<0.05$, Kolmogorov-Smirnov test), the nonparametric Wilcoxon signed rank test was used. To test equality of means of angular variables, the multisample Watson-Williams test was used. To compare paired linear data, the paired-sample $t$ test was used. To compare paired circular data, a parametric circular paired-sample test was used (built-in IGOR function). To correlate normally distributed linear-linear variables, the Pearson's correlation coefficient was used. For variables from non-normal distributions, the Spearman's rank correlation coefficient was used. The tests used in each case are specified in the text. Values are given as mean \pm SEM, unless stated otherwise. The reported $p$ values regard the Bonferroni's post hoc tests in the case of ANOVAs. All correlation coefficients are quoted as $R$.

Anatomical identification of the neurons. The recorded cells were filled with biocytin during the recordings. After the recording, the slices were fixed in $4 \%$ paraformaldehyde in $0.1 \mathrm{~m}$ phosphate buffer (PB; $\mathrm{pH} 7.4$ ) for at least $1 \mathrm{~h}$, followed by washout with $\mathrm{PB}$ several times and incubation in $30 \%$ sucrose in $0.01 \mathrm{~m} \mathrm{~PB}$ for at least $2 \mathrm{~h}$. Then slices were freeze-thawed three times above liquid nitrogen and treated with $1 \% \mathrm{H}_{2} \mathrm{O}_{2}$ in $\mathrm{PB}$ for $15 \mathrm{~min}$ to reduce the endogenous peroxidase activity. Recorded cells were visualized using the avidin-biotinylated horseradish peroxidase complex reaction (Vector Laboratories) with nickel-intensified 3,3'-diaminobenzidine as the chromogen giving a dark reaction product. After dehydration and embedding in Durcupan, cells were morphologically identified on the basis of their dendritic and axonal arborization. Representative neurons were reconstructed using a drawing tube.

\section{Results}

Gamma frequency oscillations in horizontal hippocampal slices were induced by bath application of 5-10 $\mu \mathrm{M}$ CCh. Two patch pipettes filled with aCSF were used to monitor simultaneously the LFPs in the pyramidal cell layer of hippocampal CA1 and 
CA3b regions (Fig. 1A). PSD analysis of the extracellular recordings revealed a peak in the oscillations at the same frequency in both regions (mean oscillation frequency was $31.3 \pm 0.5 \mathrm{~Hz}$ in CA3 and $31.4 \pm 0.5 \mathrm{~Hz}$ in $\mathrm{CA} 1 ; n=31 ; p=0.384$, paired sample $t$ test; Fig. $1 B, E)$. On the other hand, the power of the oscillation was always smaller in CA1 than in CA3 [572.0 (145.4-1230.0) $\mu \mathrm{V}^{2}$ in CA3 and 88.0 (27.7-196.7) $\mu \mathrm{V}^{2}$ in CA1; median and interquartile ranges are in parentheses; $n=31 ; p<0.001$, Wilcoxon signed rank test; Fig. $1 B, D]$. A strong correlation was found between the frequency of the oscillations recorded in CA3 and in CA1 ( $r=0.986, p<0.001, n=31$, Pearson's correlation, data not shown). In addition, the power of the oscillation measured in the two hippocampal regions also showed a correlation $(r=0.596, p<0.001, n=$ 31 , Spearman's rank correlation, data not shown). Cross-correlation analysis revealed a small, but significant, phase lag between the field potential oscillation recorded in the stratum pyramidale of the $\mathrm{CA} 3 \mathrm{~b}$ and CA1 regions (peak lag, $0.70 \pm$ $0.24 \mathrm{~ms} ; n=31 ; p<0.01$, one-sample $t$ test; Fig. $1 C, F)$.

After the simultaneous recording of oscillatory activities in the two hippocampal regions, the pipette from CA3 was withdrawn and loose-patch recording from a neuron was obtained, while gamma oscillation in CA1 was continuously monitored. After the recording, the spiking activity of the cell and synaptic currents from the same neuron were detected in whole-cell mode using a different pipette filled with a $\mathrm{K}^{+}$-based intrapipette solution. The intrapipette solution contained biocytin, which allowed post hoc identification of the cell types based on their morphological characteristics.

\section{Classification of the investigated cell types}

Neurons recorded in CA1 were separated into four groups: (1) CA1 pyramidal cells (CA1 PCs; $n=35$; Fig. $2 A$ ); (2) PV-eGFPpositive interneurons (PV+ INs; $n=20$; Fig. $2 B$ ); (3) oriensalveus interneurons (OA INs; $n=15$ ) with soma and dendrites located predominantly in the stratum oriens (Fig. 2C); and (4) radiatum interneurons (RAD INs; $n=14$ ) with soma and dendritic arbor mainly in the stratum radiatum (Fig. 2D). PV + INs were collected in slices prepared from PV-eGFP mice.

The group of PV + INs included basket cells, bistratified cells, and putative axo-axonic cells. These cell types have similar physiological properties in terms of firing pattern and the expression of different receptor types (Freund and Buzsáki, 1996), and we found that all PV + INs showed similar input-output properties during CCh-induced oscillations; therefore, we pooled these interneurons into a single group. Nevertheless, we cannot rule out the possibility that these cell types can play different roles in the generation or maintenance of gamma oscillations.

The group of OA INs included O-LM (oriens lacunosummoleculare) cells $(n=11)$ and O-R (oriens-radiatum) cells $(n=$ 4). The former interneurons had axonal arbors predominantly in
CA1 PC

B

$P V+I N$

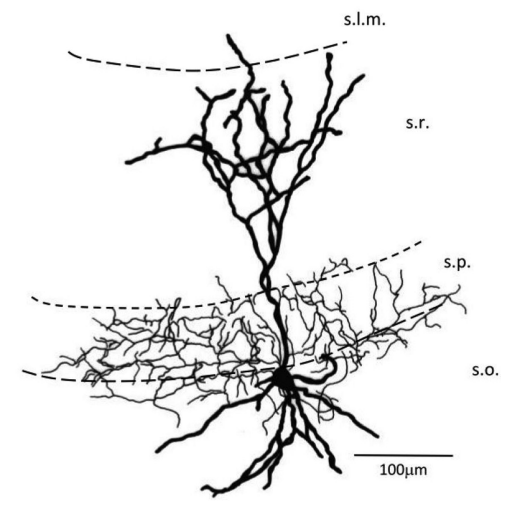

D

OA IN

RAD IN

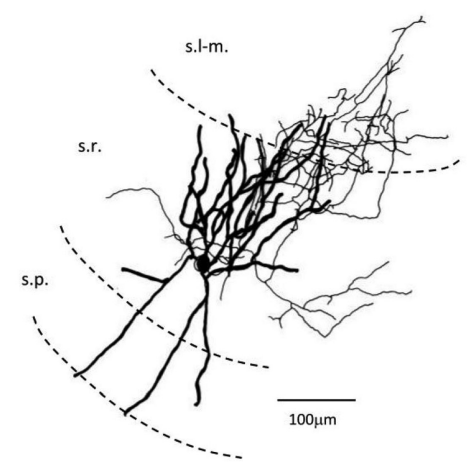

Figure 2. Light microscopic reconstructions of representative cells of the investigated cell groups recorded in the CA1 region of the hippocampus. $A, A C A 1 P C ; B$, a parvalbumin-expressing basket cell (PV+ IN); $C$, an OA IN; $D$, a RAD IN. Dendrites are represented in black and axons in gray. s.I.m., Stratum lacunosum-moleculare; s.r., stratum radiatum; s.p., stratum pyramidale; s.o., stratum oriens.

the stratum lacunosum-moleculare (McBain et al., 1994), whereas the latter cells projected their axon collaterals into the strata oriens and radiatum as well as toward the subiculum (Zemankovics et al., 2010). The OA INs are typically considered as feedback inhibitory cells, since their main excitatory input originates from CA1 PCs (Blasco-Ibáñez and Freund, 1995).

The group of RAD INs included several different cell types, such as radiatum-lacunosum-moleculare cells $(n=3)$, neurogliaform cells $(n=2)$, Schaffer collateral-associated cells $(n=4)$, and subiculum-projecting GABAergic cells $(n=5)$ (Somogyi and Klausberger, 2005). Although the recorded neuron types formed a diverse cell population based on their morphological features, they are usually referred to as feedforward inhibitory cells because their main excitatory intrahippocampal input is formed by the Schaffer collaterals of the CA3 PCs (Li et al., 1994).

In addition to CA1 neurons, we also obtained recordings from CA3 pyramidal cells (CA3 PCs; $n=22$ ) and perisomatic regiontargeting CA3 interneurons (CA3 PTIs; $n=10$ ) during CChinduced oscillations monitored in CA1. The group of CA3 PTIs contained interneurons with axon arborization in the somatic and proximal dendritic region of CA3 PCs. Seven of the 10 recorded CA3 PTIs were also characterized as PV+ INs. As shown in previous studies, CA3 PTIs fire at the ascending phase of the oscillation with a monosynaptic delay after the firing of CA3 PCs, which excitatory neurons spike at the trough of the oscillation cycle (Hájos et al., 2004; Gulyás et al., 2010). 
Table 1. Firing properties of the different cell types during $\mathrm{CCh}$-induced gamma oscillations

\begin{tabular}{lrlll}
\hline & Rate (Hz) & $\begin{array}{l}\text { Spike frequency/ } \\
\text { 0scillation frequency }\end{array}$ & \multicolumn{1}{l}{ AP } & \multicolumn{1}{c}{$\Phi_{\text {AP }}($ rad $)$} \\
\hline CA1 PCs $(n=15)$ & $11.07 \pm 1.35$ & $0.33 \pm 0.04$ & $0.21 \pm 0.02$ & $-2.25 \pm 0.23$ \\
PV + INs $(n=11)$ & $15.65 \pm 2.18$ & $0.54 \pm 0.08$ & $0.75 \pm 0.04$ & $-1.13 \pm 0.06$ \\
OA INs $(n=15)$ & $24.79 \pm 3.37$ & $0.78 \pm 0.11$ & $0.49 \pm 0.07$ & $-1.22 \pm 0.07$ \\
RAD INs $(n=11)$ & $8.71 \pm 1.46$ & $0.28 \pm 0.05$ & $0.36 \pm 0.06$ & $-1.03 \pm 0.37$ \\
CA3 PCs $(n=22)$ & $4.44 \pm 0.46$ & $0.14 \pm 0.01$ & $0.54 \pm 0.03$ & $-1.72 \pm 0.04$ \\
CA3 PTIs $(n=10)$ & $28.38 \pm 6.31$ & $1.15 \pm 0.37$ & $0.70 \pm 0.06$ & $-1.23 \pm 0.13$ \\
\hline
\end{tabular}

Data are presented as mean \pm SEM. The dataset contains only phase-coupled cells.

frequency of CA1 PCs, RAD INs, and CA3 PCs (CA3 PCs vs CA1 PCs, $p=0.624$; CA3 PCs vs CA1 RAD INs, $p=1$; CA1 PCs vs RAD INs, $p=1$; ANOVA with Bonferroni's post hoc test). Some of the OA INs and CA3 PTIs fired doublets of action potentials during numerous gamma cycles, which resulted in a rather high mean firing rate of these groups, on average. RAD INs fired at the lowest rates among INs; however, the difference in firing rates reached significance only in comparison with OA INs and CA3 PTIs ( $p<0.001$ in both cases, ANOVA with Bonferroni's post hoc test; Figs. $3 B, E, 4 A, B$; Table 1 ).

Most of the recorded neurons showed gamma-modulated firing according to the Rayleigh test $\left(p_{\mathrm{r}}<\right.$ 0.01 ), only 6 of 21 CA1 PCs and 3 of 14 RAD INs were not significantly phasecoupled to the ongoing network oscillation detected in CA1. There were no significant differences in the firing rate of phase-coupled and nonphase-coupled cells within a cell group. The firing rate was $11.07 \pm 1.35 \mathrm{~Hz}$ for phase-coupled CA1 PCs $(n=15)$ and $9.26 \pm 2.52 \mathrm{~Hz}$ for nonphase-coupled PCs $(n=6, p=0.624)$ and was $8.71 \pm 1.42 \mathrm{~Hz}$ for phase-coupled RAD INs, $(n=11)$ and $8.18 \pm 0.83 \mathrm{~Hz}$ for nonphase-coupled RAD INs $(n=3 ; p=$ 0.855 , Student's $t$ test).

Although the firing of almost all of the recorded neurons was modulated by the ongoing field oscillation, there were some significant differences in the depth of modulation among the cell types. CA1 PCs $(n=15)$ were significantly less phasecoupled than PV + INs $(n=11, p<$ $0.001)$, OA INs $(n=15, p<0.001)$, CA3 PCs $(n=22, p<0.001)$, and CA3 PTIs $(n=10, p<0.001)$. Whereas CA1 PCs were significantly phase-coupled, the spike phase preference was broadly tuned across the cycle (Figs. 3C, 4D). We did not find a significant difference in the strength of phase-coupling $\left(r_{\mathrm{AP}}\right)$ between CA1 PCs and CA1 RAD INs $(n=11, p=0.5584)$. Among CAl cells, the firing of PV+ INs was the most precisely phase-coupled to the ongoing field potential oscillation. Besides the mentioned differences in the coupling strength of CA1 PCs and PV+ INs, the $r_{\mathrm{AP}}$ of the latter cell type was significantly higher than the $r_{\mathrm{AP}}$ of OA INs $(p<0.01)$, RAD INs $(p<0.001)$, and CA3 PCs $(p<0.05)$. How-

Except for CA3 pyramidal cells, only cells that could be unequivocally classified into one of these categories based on their morphological features were included in the study. In the case of CA3 PCs, data of spiking properties of cells identified only by visual inspection were also included in the analysis.

\section{Firing properties of different cell types during $\mathrm{CCh}$-induced} network oscillation in CA1

Comparison of the firing frequency between cell types (Table 1) revealed that CA3 PCs tended to fire at lower rates than the other cell types; they fired at significantly lower frequencies than $\mathrm{PV}+$ INs $(p<0.05)$, OA INs $(p<0.001)$, and CA3 PTIs $(p<0.001)$. However, there was no significant difference between the firing ever, there were no significant differences between the phasecoupling strength of CA3 PTIs and PV+ INs $(p=1$, ANOVA with Bonferroni's post hoc test; Figs. 3C,F, 4C-E; Table 1).

Despite the fact that most of the recorded neurons fired phasecoupled, they did not all fire at the same phase of the gamma cycle. There were systematic differences in the preferred phase $\left(\Phi_{\mathrm{AP}}\right)$ of the different cell types. CA1 PCs were less likely to fire around the peak of the local oscillation, whereas both CA1 and CA3 INs tended to fire on the ascending phase of the cycle. There were no significant differences in the preferred phase of different IN types in CA1 (PV+ INs vs OA INs, $p=0.295 ; \mathrm{PV}+$ INs vs RAD INs, $p=0.778$; OA INs vs RAD INs, $p=0.538$ ) or between CA1 INs and CA3 PTIs (PV+ INs vs CA3 PTIs, $p=0.408$; OA INs 
vs CA3 PTIs, $p=0.9539$; RAD INs vs CA3 PTIs, $p=0.5936)$. CA3 PCs also fired near the trough, but significantly later than the preferred phase of CA1 PCs $(p<0.01)$ and significantly earlier than INs within a gamma cycle (where the cycle starts at $-\pi$ and ends at $+\pi$ by definition; CA1 PCs vs PV + INs, $p<0.001$; CA1 PCs vs OA INs, $p<0.001$; CA1 PCs vs RAD INs, $p<0.01$; CA1 PCs vs CA3 PTIs, $p<0.01$, WatsonWilliams test). When translating these phase differences to time differences according to the mean oscillation frequency $(31 \mathrm{~Hz})$, we found that the time difference was $\sim 3$ ms between the discharge of CA3 PCs and INs of both regions, 5-6 ms between the spiking of CA1 PCs and INs, and 1-2 ms between the firing of CA1 PCs and CA3 PCs within a gamma cycle (Figs. $3 C, F, 4 C-E$; Table 1).

In summary, we found that CA1 and CA3 PCs showed distinct behavior during oscillations. While CA3 PCs fired with high precision close to the trough of the gamma cycle, CA1 PCs did not show a clear peak in their firing probability, but their firing was not randomly distributed over a cycle. Meanwhile, CA3 and CA1 INs showed a similar firing characteristic during the oscillation: both groups fired strongly phase-coupled at the ascending phase of the oscillation (except some CA1 RAD INs). The sequence in the discharge of the different cell types suggests that during CCh-induced oscillations, CA3 PCs could excite both CA3 and CA1 INs that fire at latencies indicative of monosynaptic connections, but the firing of CA1 PCs was not driven by the discharge of CA3 PCs. These data suggest that oscillations generated in CA3 may propagate to CA1 through the direct discharge of CA1 INs driven by CA3 PC spiking.

Characteristics of synaptic inputs in the different cell types during $\mathrm{CCh}$-induced gamma oscillation in CA1

To test the hypothesis that gamma oscillations propagate from CA3 to CA1 via feedforward inhibition, we asked what determines the firing properties of the different cell types during oscillations. To answer this question, we recorded the excitatory and inhibitory postsynaptic currents of the same cells during oscillations and related them to the firing activity of the neurons. EPSCs were recorded at the estimated reversal potential of IPSCs (approximately $-70 \mathrm{mV}$ ), whereas IPSCs were recorded at the estimated reversal potential of EPSCs $(\sim 0 \mathrm{mV})$. To characterize the postsynaptic currents in a neuron, we determined the phase-coupling strength of EPSCs and IPSCs $\left(r_{\mathrm{e}}\right.$ and $r_{\mathrm{i}}$, respectively), as well as the phasic excitatory and inhibitory charge transfer. To define the phase-coupling strength of the postsynaptic currents, the phase of the peak current (i.e., the peak amplitude) recorded in each cycle was calculated. The com-
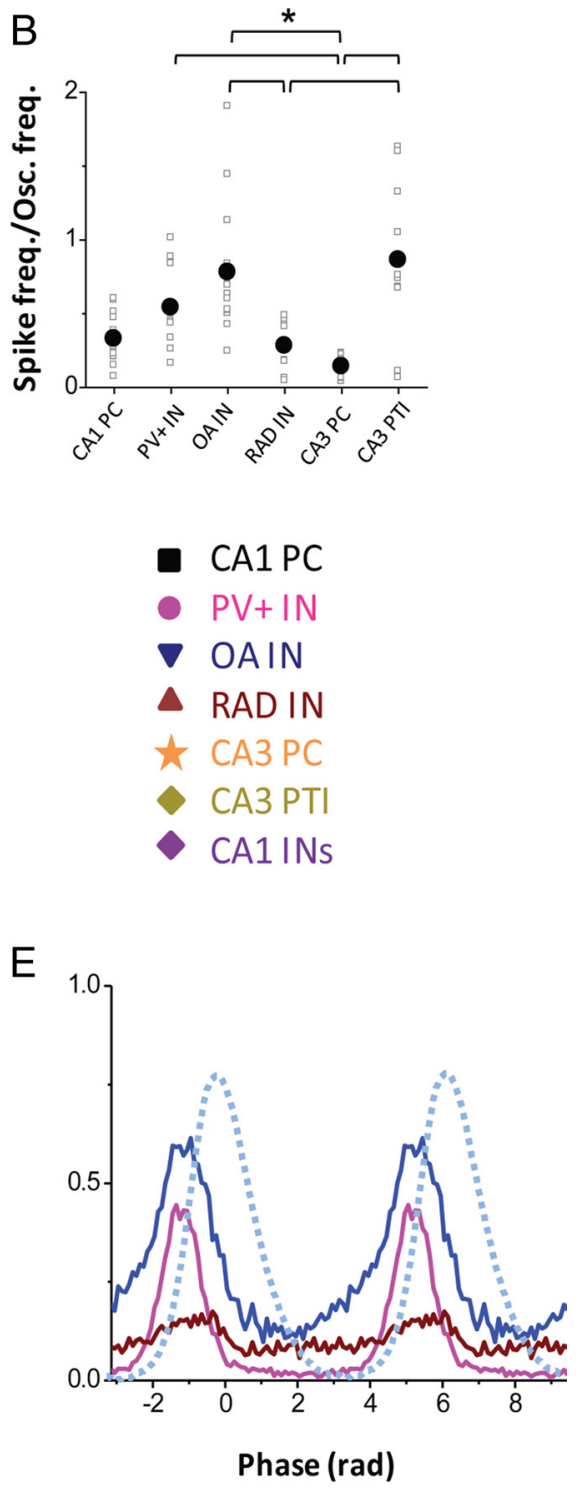

Figure 4. Firing properties of the different cell types during $C(h$-induced gamma oscillations. $\boldsymbol{A}$, Firing frequency of the different neuron types. Phase-coupled cells $\left(p_{\mathrm{r}}<0.01\right)$ are indicated with open symbols and nonphase-coupled cells with solid gray symbols. Means are indicated with black dots. $\boldsymbol{B}$, The normalized spiking frequency of the phase-coupled cells of the different cell groups. $C$, The phase-coupling strength of firing $\left(r_{A P}\right)$ for each phase-coupled neuron is plotted as a function of the mean gamma phase. D, The probability of discharge for CA1 PCs, CA3 PCs, CA1 INs, and CA3 INs as a function of a gamma cycle. Here the data from all CA1 INs were pooled. Note that CA1 PCs tended to fire at the trough of the oscillation, CA3 PCs fired somewhat later, and both 1 INs fired mainly at the ascending phase of the oscillation. $\boldsymbol{E}$, As in $\boldsymbol{D}$, but here the spiking probabilities of distinct CA1 IN types are shown separately. Different symbols and colors mark different cell types as indicated in the inset. The dotted blue line shows the cycle-averaged field oscillation. Asterisks indicate the significant differences.

parison of synaptic inputs in the different cell types and the correlation analyses of synaptic inputs and firing properties were performed only on those cells that showed significant gamma modulation in their spiking activity.

We found that both EPSCs and IPSCs were strongly phasecoupled in all neuron types and that there was no difference in the strength of the phase-coupling of postsynaptic currents between the different cell types (Figs. 5C,F, 6C,F, $7 D$, E; Table 2). However, there were significant differences in the amount of phasic charge transfer. CA3 PTIs $(n=5)$ received the largest phasic excitatory synaptic input among all cell types, whereas CA1 PCs $(n=11)$ received the smallest. Among CA1 cells, the largest phasic excitatory charge transfer could be measured in PV+ INs $(n=9)$. Among CA1 interneurons, RAD INs $(n=7)$ received the 
CA1 PC

A

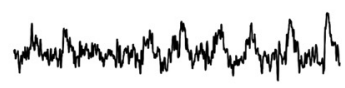

B

C

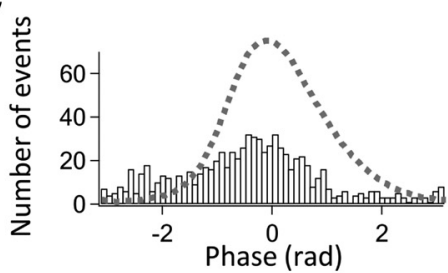

D

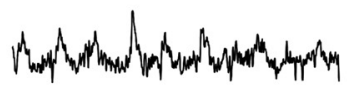

F
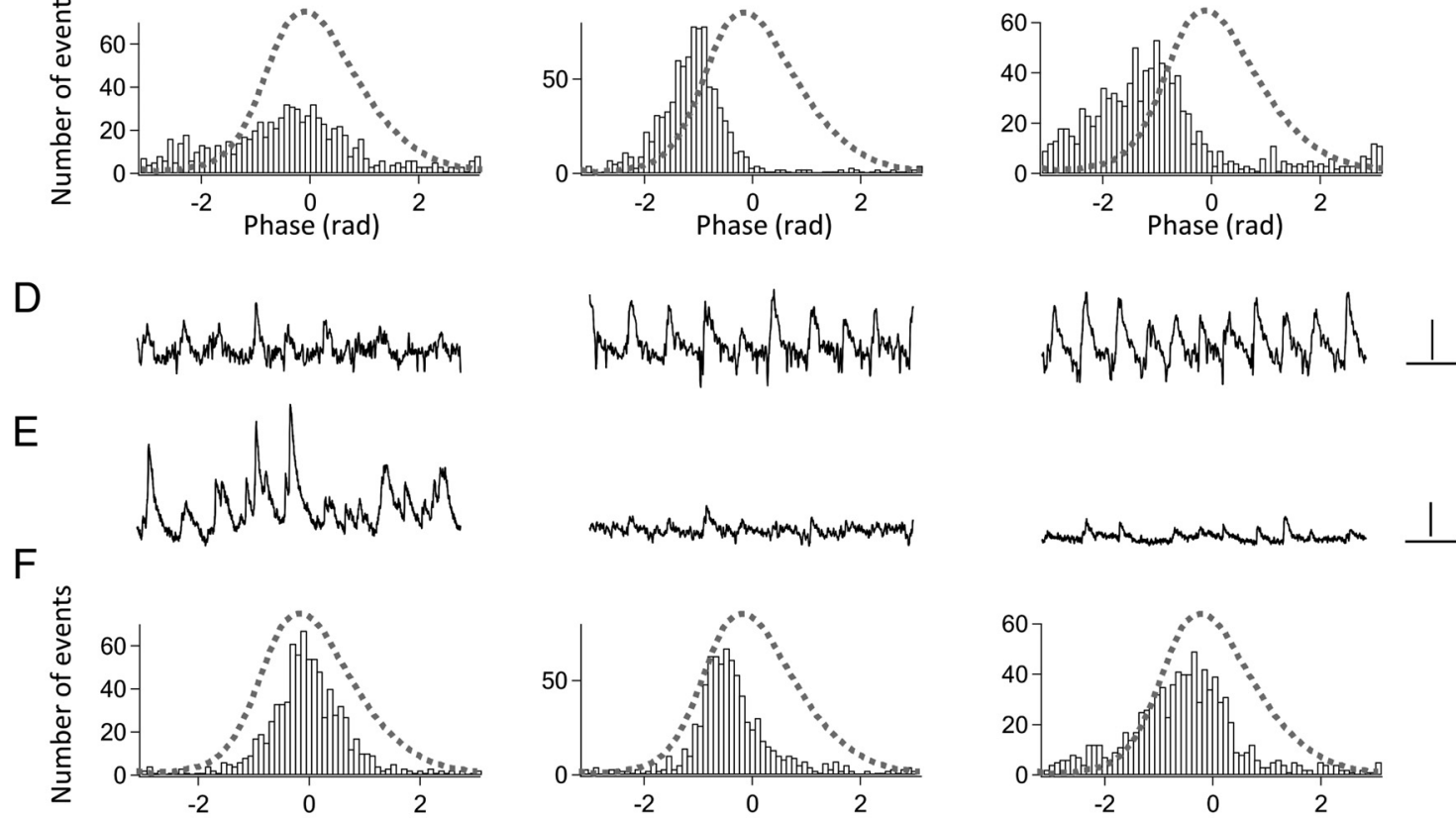

G
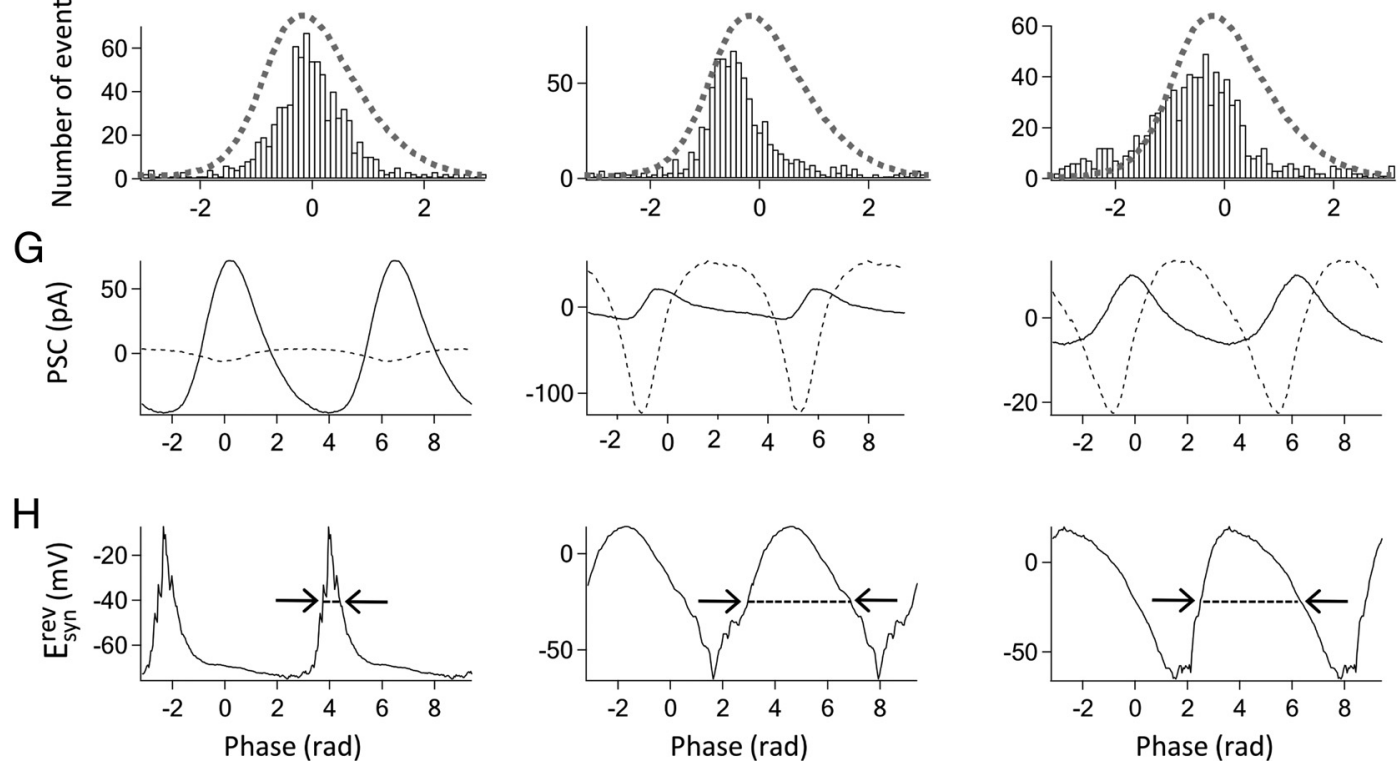

Figure 5. Postsynaptic currents of the different cell types during CCh-induced oscillations I. $A, B$, Simultaneous recordings of field potential oscillations in the stratum pyramidale of CA1 ( $A$ ) and EPSCS recorded from a representative CAI PC, PV + IN, and OA IN (B) (the same cells as in Fig.3). EPSCs were recorded in whole-cell voltage-clamp mode at the estimated reversal potential of IPSCs (approximately - 70 $\mathrm{mV})$. C, The EPSC-phase histogram of the cells showing the distribution of the EPSC peak amplitudes during a 30-s-long recording epoch. $\boldsymbol{D}-\boldsymbol{F}$, The same as in $\boldsymbol{A}-\boldsymbol{C}$, but for IPSCs that were recorded at the estimated reversal potential of EPSCS $\sim 0 \mathrm{mV})$. The dotted lines in Cand F indicate the average gamma cycle. G, Cycle-averaged PSCsin the given CA1 PCs, PV+ INs, and OAINs. Dotted line, EPSC; solid line, IPSC. $\boldsymbol{H}$, The net apparent synaptic reversal potential $\left(E_{s y n}^{r e v}\right)$ as a function of an oscillation cycle. Arrows indicate the half-width of the $E_{s y n}^{r e v}$ curve. Note the differences in the half-width of $E_{s y n}^{r e v}$ curve between PCs and most of the INs (see also Fig. 6). Calibration for field potential traces: vertical, $0.1 \mathrm{mV}$; horizontal, $0.05 \mathrm{~s}$. Calibration for current traces: vertical, $100 \mathrm{pA}$; horizontal, $0.05 \mathrm{~s}$.

smallest phasic excitation. Significant differences in phasic excitatory charge $\left(Q_{\mathrm{e}}\right)$ could be detected between $\mathrm{PV}+\mathrm{INs}$ and CA1 PCs $(p<0.05)$, CA3 PTIs and CA1 PCs $(p<0.001)$, CA3 PTIs and RAD INs $(p<0.001)$, CA3 PTIs and OA INs $(n=6, p<$ $0.05)$, and CA3 PTIs and CA3 PCs $(n=6, p<0.05$, ANOVA with Bonferroni's post hoc test) (Figs. $5 B, C, G, 6 B, C, G$, $7 A$; Table 2).

Phasic inhibitory charge transfer was significantly larger in CA3 PCs than in all other cell types except CA3 PTIs $(p<0.001$ between CA3 PCs and all CA1 cell groups, whereas $p=0.09$ when comparing CA3 PTIs and CA3 PCs). There was no difference in the amount of phasic inhibition between cell types within CA1 ( $p=1$ in all comparisons) or CA1 cells and CA3 PTIs ( $p>0.25$ in all comparisons, ANOVA with Bonferroni's post hoc test; Fig. $5 E-G, 6 E-G, 7 B$; Table 2).

To investigate the relationship between somatically recorded synaptic excitation and inhibition within a cell type, we 


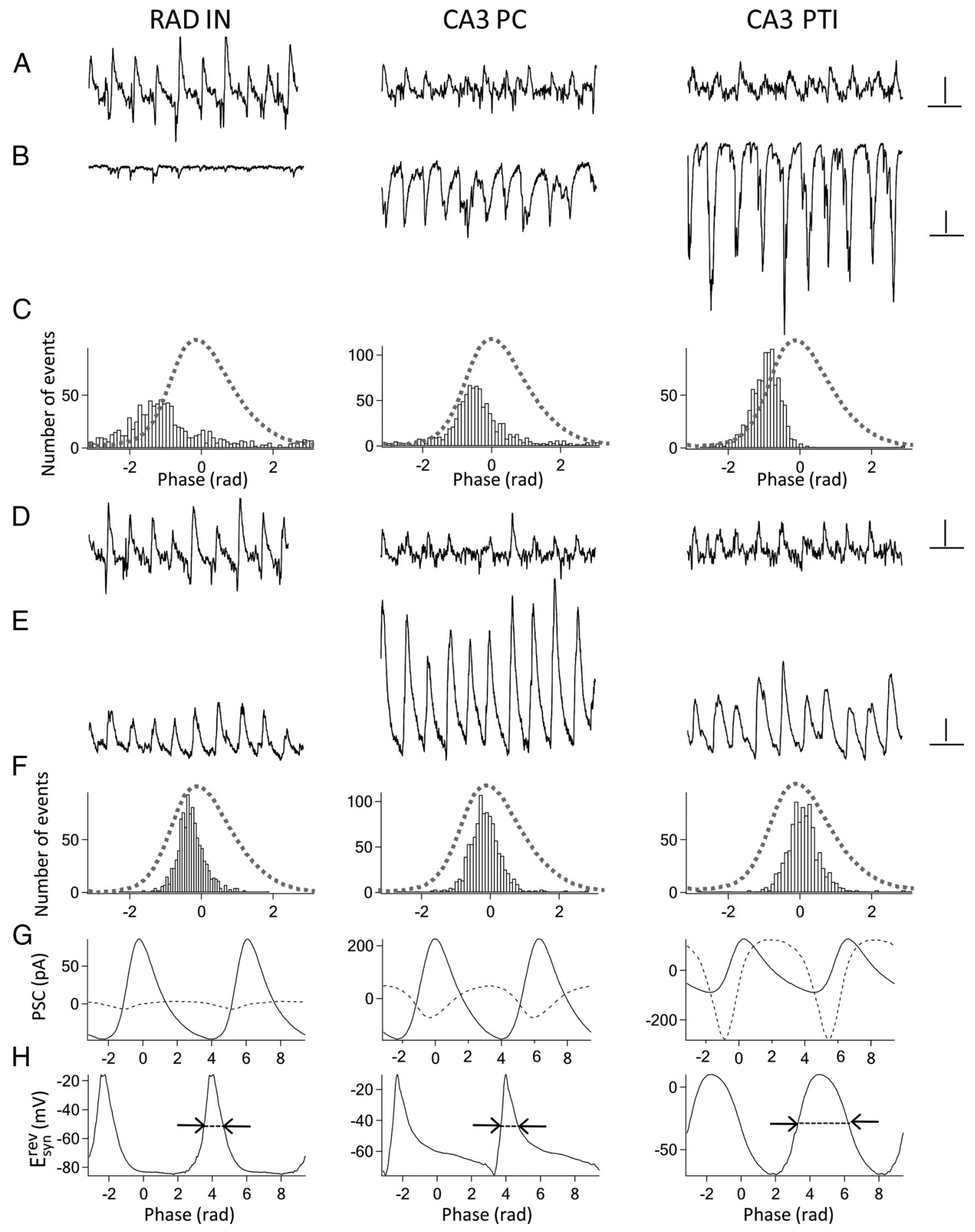

Figure 6. Postsynaptic currents of the different cell types during CCh-induced oscillations II. $A, B$, Simultaneous recordings of field potential oscillations in the stratum pyramidale of $C A 1(A)$ and EPSCs recorded from a representative RAD IN, CA3 PC, and CA3 PTI (B; RAD IN is the same cell as in Fig. 3). EPSCs were recorded in whole-cell voltage clamp mode at the estimated reversal potential of IPSCS (approximately $-70 \mathrm{mV}$ ). C, The EPSC-phase histogram of the cells showing the distribution of the EPSC peak amplitudes during a 30-s-long recording epoch. $\boldsymbol{D}-\boldsymbol{F}$, The same as in $\boldsymbol{A}-\boldsymbol{C}$, but for IPSCs that were recorded at the estimated reversal potential of EPSCS $(\sim 0 \mathrm{mV})$. Dotted lines in (and $F$ indicate the average gamma cycle. G, Cycle averaged PSCs in the given RAD IN, CA3 PC, and CA3 PTI. Dotted line, EPSC; solid line, IPSC. $\boldsymbol{H}$, The net apparent synaptic reversal potential $\left(E_{s y n}^{r e v}\right)$ as a function of an oscillation cycle. Arrows indicate the half-width of the $E_{s y n}^{\text {rev }}$ curve. Note the differences in the half-width of the $E_{s y n}^{r e v}$ curve between PC and most of the INs (see also Fig. 5). Calibration for field potential traces: vertical, $0.1 \mathrm{mV}$; horizontal, 0.05 s. Calibration for current traces: vertical, $100 \mathrm{pA}$; horizontal, $0.05 \mathrm{~s}$.

calculated the ratio of phasic excitatory-to-inhibitory charge $\left(Q_{e} / Q_{i}\right)$. Phasic inhibition exceeded phasic excitation in all PCs, in both CA1 and CA3, and also in some RAD INs, whereas the dominant input was excitatory in most of the INs in both regions. $Q_{\mathrm{e}} / Q_{\mathrm{i}}$ was significantly smaller in CA1 PCs than PV+ INs $(p<0.001)$ and CA3 PTIs $(p<0.05)$. CA3 PCs and RAD INs also had a significantly smaller $Q_{\mathrm{e}} / Q_{\mathrm{i}}$ ratio than $\mathrm{PV}+$ INs (CA3 PCs vs PV+ INs, $p<0.001$; RAD INs vs CA1 $\mathrm{PV}+\mathrm{INs}, p<0.01$; ANOVA with Bonferroni's post hoc test; Figs. 5G, 6G, 7C; Table 2). 

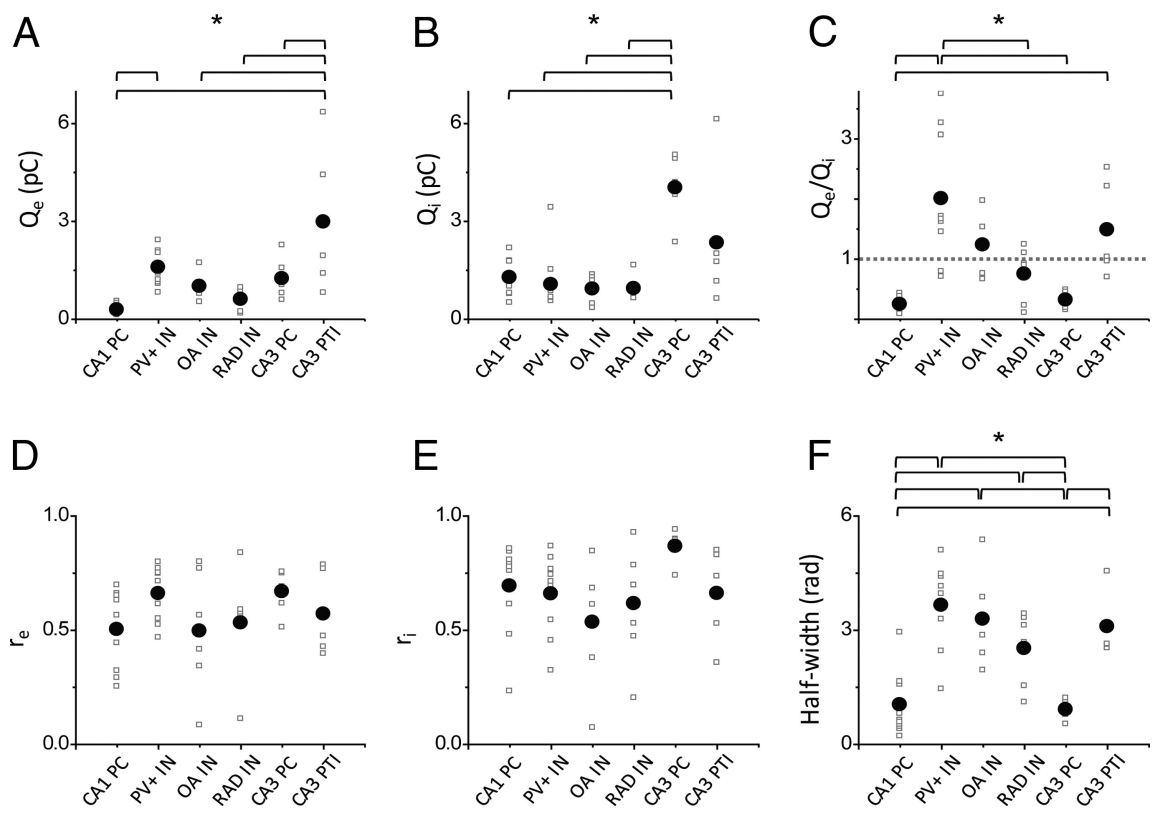

Figure 7. Properties of the postsynaptic currents measured in the different cell types. $\boldsymbol{A}, \boldsymbol{B}$, Phasic excitatory $\left(Q_{\mathrm{e}} ; \boldsymbol{A}\right)$ and inhibitory $\left(Q_{j} ; B\right)$ charge transfer in the different neuron classes. The differences in $Q_{e}$ reached significance between CA1 PCs and PV + INs $(p<0.001)$ and between CA1 PCs and CA3 INs $(p<0.001)$, RAD INs and CA3 INs $(p<0.001)$, and OA INs and CA3 INs $(p<0.05)$, whereas CA3 PCs had a larger $Q_{i}$ than all other cell types $(p<0.001$ in all comparisons between CA3 $P C s$ and other cell types). C, Phasic excitatory/inhibitory charge ratio $\left(Q_{\mathrm{e}} / Q_{i}\right)$ in the different cell types. CA1 PCs had smaller $Q_{\mathrm{e}} / Q_{i}$ than $P V+I N s(p<$ $0.001), 0 A$ INs $(p<0.05)$, and CA3 INs $(p<0.01)$, and the difference between $Q_{\mathrm{e}} / Q_{i}$ also reached significance between PV + INs and RAD INs $(p<0.05)$ and between PV + INs and CA3 PCs $(p<0.001) \cdot \boldsymbol{D}, \boldsymbol{E}$, The phase-coupling strength of the peak EPSCs $\left(r_{\mathrm{e}}\right.$; D) and IPSCs $\left(r_{\mathrm{i}} ; \boldsymbol{E}\right)$ in the different cell types. $\boldsymbol{F}$, The half-width of the $E_{s y n}^{r e v}$ curve of the different cell groups. The half-width was significantly smaller in both CA1 and CA3 PCs than in the INs ( $p<0.05$ in all comparisons between PC and IN pairs). Means are indicated as black dots, whereas asterisks mark the significant differences (ANOVA with Bonferroni's post hoc test).

In all neurons, the phase of the peak excitation preceded the phase of the peak inhibition. The peak excitation occurred between -1.5 and -0.6 radians, on the ascending phase of the field oscillation, whereas the peak inhibition was detected always somewhat later between -0.6 and -0.2 radians, closer to the peak of the field oscillation. Comparison of the mean phases of the EPSC peak amplitudes revealed significant differences between PCs and INs. The phase of synaptic excitation was significantly later in both CA1 PCs and CA3 PCs than in INs of both areas ( $p<0.01$ in all comparisons). On the other hand, the mean phase of the IPSC peak amplitude was, on average, somewhat later in PV+ INs and OA INs than in CA1 PCs, CA3 PCs, and RAD INs ( $p<0.05$ in all of these comparisons, Watson-Williams test) (Figs. 5F, 6F, see Figs. 10, 11; Table 2).

We next determined the combined effect of synaptic input arriving at the soma. To capture the temporal relationship between inhibitory and excitatory synaptic conductances, we calculated the net apparent synaptic reversal potential $E_{s y n}^{r e v}$ (see Materials and Methods). $E_{s y n}^{r e v}$ describes the effective synaptic conductance during a cycle and consequently provides a measure of the balance of excitation and inhibition. There were clear differences in the shape of the $E_{s y n}^{r e v}$ curve depending on the cell type. In general, the half-width of the $E_{s y n}^{r e v}$ curve was significantly narrower in PCs in both CA1 and CA3 than in INs of both regions $(p<0.05$ in all comparisons except CA3 PCs vs RAD INs, where $p=0.079$; ANOVA with Bonferroni's post hoc test) reflecting the dominant inhibitory input received by PCs and the prevailing excitatory input received by interneurons during the oscillation (Figs. 5H, 6H, 7F; Table 2).
Correlations between firing properties and synaptic currents in the different cell types

After establishing both the input and output characteristics of different cell types, we sought to determine input-output relationships in the phase-coupled cells. As $\mathrm{PV}+\mathrm{INs}$ and $\mathrm{OA}$ INs fired at higher rates than CA1 PCs and RAD INs, and the phasic excitatory charge transfer was also larger in these cells, we asked whether the firing rate of cells may be correlated with excitatory charge transfer. Indeed, when comparing these quantities over all CA1 cells, we found a positive correlation between $Q_{\mathrm{e}}$ and the firing frequency $(r=$ $0.495, p<0.01, n=33$; Fig. $8 A$ ). Interestingly, no correlation could be found between the excitatory charge transfer and the firing rate, when it was tested for CA1 PCs only (data not shown, $r=0.284, p=$ $0.371, n=11$ ), but the correlation between $Q_{e}$ and firing frequency reached significance, when it was tested on CA1 INs (data not shown, $r=0.437, p<0.05$, $n=22)$. In addition, the ratio of excitatory and inhibitory charge $\left(Q_{\mathrm{e}} / Q_{\mathrm{i}}\right)$ also correlated with the firing rate over all CA1 cells $(r=0.401, p<0.05, n=33$; Fig. $8 C)$. In contrast, phasic inhibitory charge did not correlate with firing frequency $(r=$ $0.063, p=0.728, n=33$, Pearson's correlation analyses; Fig. $8 B$ ). These data support the hypothesis that excitatory synaptic input controls the firing rate of the CAl inhibitory cells.

In the case of INs, not only the firing rate, but also the phasecoupling strength, correlated with the excitatory input. There was a positive correlation between both $r_{\mathrm{e}}$ and $r_{\mathrm{AP}}(r=0.675, p<$ $0.001, n=22)$, and $Q_{\mathrm{e}}$ and $r_{\mathrm{AP}}$ among CA1 INs $(r=0.664, p<$ $0.001, n=22$; Fig. $9 A, C$ ). No correlation could be observed between $r_{\mathrm{i}}$ and $r_{\mathrm{AP}}(r=0.326, p=0.138, n=22)$ or between $Q_{\mathrm{i}}$ and $r_{\mathrm{AP}}(r=0.255, p=0.251, n=22$; Fig. $9 B, D)$. However, in the case of CA1 PCs, which tended to show lower phase-coupling than INs, we could not find any correlations between $r_{\mathrm{AP}}$ and their synaptic inputs $\left(r_{\mathrm{AP}}\right.$ and $r_{\mathrm{e}}: r=0.359, p=0.278 ; r_{\mathrm{AP}}$ and $Q_{\mathrm{e}}$ : $r=0.357, p=0.281 ; r_{\mathrm{AP}}$ and $r_{\mathrm{i}}: r=0.147, p=0.666 ; r_{\mathrm{AP}}$ and $Q_{\mathrm{i}}$ : $r=0.255, p=0.251 ; n=11$, Pearson's correlation analyses; Fig. $9 E-H)$.

These analyses reveal that precise and robust excitatory input received by CA1 INs was associated with precise firing. In contrast, no such correlation could be observed in the case of CA1 PCs. In summary, these results also support the hypothesis that the firing of INs is primarily driven by their excitatory synaptic inputs. Although CA1 PCs receive their excitatory input with equally high temporal precision, the synaptic excitatory charge transfer appears insufficient to control spike timing in these cells during CCh-induced network oscillation.

\section{Phase and time relationships between firing and synaptic inputs in the different cell types}

We also compared the phase of the analyzed events (Fig. 10; Tables 1, 2). We found that for PV+ INs, OA INs, most RAD INs, and all CA3 PTIs, the phase of action potentials showed a close 
Table 2. The properties of synaptic inputs of the different cell types during CCh-induced gamma oscillations

\begin{tabular}{|c|c|c|c|c|c|c|c|c|}
\hline & $r_{\mathrm{e}}$ & $r_{\mathrm{i}}$ & $Q_{e}(p C)$ & $Q_{i}(p c)$ & $Q_{e} / Q_{i}$ & $\Phi_{\mathrm{e}}(\mathrm{rad})$ & $\Phi_{\mathrm{i}}(\mathrm{rad})$ & $E_{s y n}^{r e v} \mathrm{hw}(\mathrm{rad})$ \\
\hline $\operatorname{CA} 1 \mathrm{PCs}(n=11)$ & $0.5 \pm 0.05$ & $0.69 \pm 0.06$ & $0.29 \pm 0.04$ & $1.27 \pm 0.15$ & $0.24 \pm 0.04$ & $-0.64 \pm 0.15$ & $-0.18 \pm 0.12$ & $1.04 \pm 0.25$ \\
\hline $\mathrm{PV}+\operatorname{INs}(n=9)$ & $0.66 \pm 0.04$ & $0.66 \pm 0.06$ & $1.59 \pm 0.19$ & $1.06 \pm 0.31$ & $2.00 \pm 0.36$ & $-1.16 \pm 0.11$ & $-0.62 \pm 0.12$ & $3.65 \pm 0.37$ \\
\hline OA INs $(n=6)$ & $0.50 \pm 0.11$ & $0.53 \pm 0.11$ & $1.01 \pm 0.16$ & $0.92 \pm 0.18$ & $1.23 \pm 0.20$ & $-1.52 \pm 0.08$ & $-0.54 \pm 0.08$ & $3.29 \pm 0.5$ \\
\hline RAD INs $(n=7)$ & $0.53 \pm 0.08$ & $0.62 \pm 0.09$ & $0.6 \pm 0.11$ & $0.94 \pm 0.13$ & $0.75 \pm 0.16$ & $-1.23 \pm 0.15$ & $-0.19 \pm 0.14$ & $2.52 \pm 0.34$ \\
\hline CA3 PCs $(n=6)$ & $0.67 \pm 0.04$ & $0.86 \pm 0.03$ & $1.24 \pm 0.25$ & $4.02 \pm 0.40$ & $0.32 \pm 0.06$ & $-0.66 \pm 0.09$ & $-0.23 \pm 0.14$ & $0.91 \pm 0.11$ \\
\hline CA3 PTIs $(n=5)$ & $0.57 \pm 0.09$ & $0.66 \pm 0.09$ & $2.98 \pm 1.04$ & $2-33 \pm 0.98$ & $1.49 \pm 0.37$ & $-1.32 \pm 0.17$ & $-0.41 \pm 0.18$ & $3.09 \pm 0.38$ \\
\hline
\end{tabular}

Data are presented as mean \pm SEM. The dataset contains only those cells that fired phase-coupled to the ongoing field oscillation in CA1 and also fulfilled the requirements to be included in the voltage-clamp dataset (see Materials and Methods). hw, Half-width.
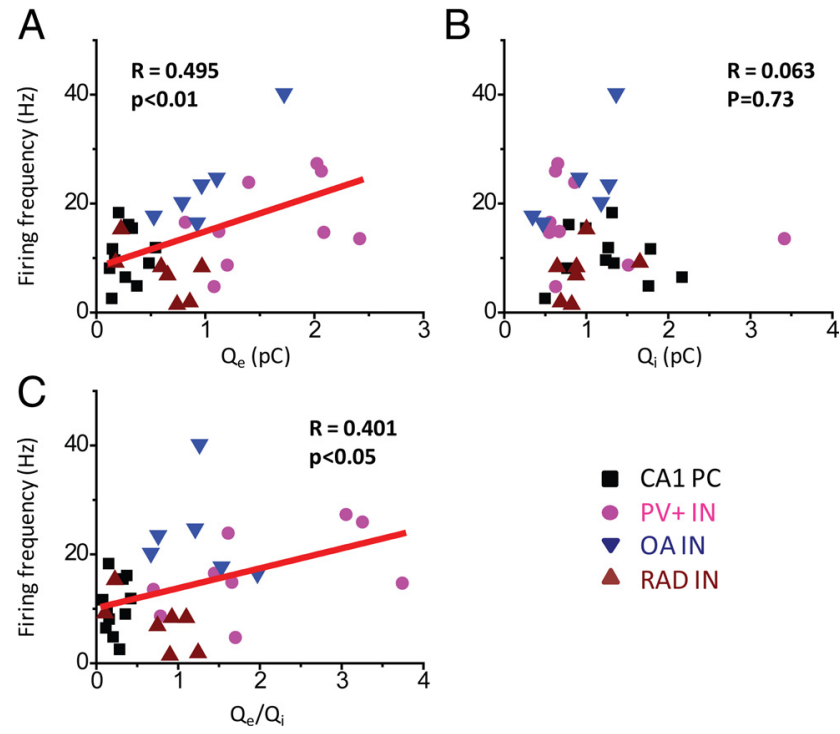

Figure 8. Correlations between the firing frequency and the phasic synaptic charge transfer among $C A 1$ cells. The firing frequency plotted against phasic excitatory $\left(Q_{j} ; A\right)$ and inhibitory $\left(Q_{j} ; B\right)$ charge transfer and phasic excitatory/inhibitory charge transfer ratio $\left(Q_{e} / Q_{i} ; C\right)$ for individual CA1 cells. Significant correlations were found between the firing rate and $Q_{e}$ and the firing rate and $Q_{e} / Q_{j}$. Different cell types are marked with different symbols (Pearson's correlation).

coincidence with the phase of the EPSC peak (either slightly preceding or following the peak). However, the phase of action potentials occurred much earlier in CA1 PCs $(p<0.01)$ and in some RAD INs than the phase of peak excitation ( $>8 \mathrm{~ms}$ earlier according to the mean oscillation frequency of $31 \mathrm{~Hz}$ ). CA3 PCs also fired significantly earlier than their peak excitatory input $(p<$ 0.001 ), but still later within the cycle than CA1 PCs (2-3 ms later). The phase of peak inhibition always occurred later in a cycle than the phase of the action potentials in all recorded cells independent of the cell type or the region (always 2-5 ms later than the peak excitation in a given cell).

\section{The firing of CA1 INs is driven by CA3 excitatory input}

Together, these data suggest that during CCh-induced oscillations, the firing of CA1 INs is driven by CA3 PCs, whereas the discharge of CA1 PCs is not controlled by their main intrahippocampal excitatory drive but, rather, by the recruited inhibition. This model leads to the testable prediction that blocking inhibition locally in CA1 should not significantly change the firing activity of CA1 INs, consistent with the feedforward inhibitory model of the propagation of gamma oscillation from CA3 to CA1. PC firing may or may not be affected by local GABA receptor antagonism, depending on the location of the inhibitory synapses relative to the puff.
We applied a $\mathrm{GABA}_{\mathrm{A}}$ receptor antagonist, SR-95531 (gabazine, $50 \mu \mathrm{M}$ ), locally onto the stratum pyramidale of the CA1 region. Since $\mathrm{GABA}_{\mathrm{A}}$ receptor-mediated postsynaptic currents underlie the generation of CCh-induced gamma oscillation in slices (Oren et al., 2010), we expected that puffing gabazine into CA1 would eliminate the field oscillation in this area. At the same time, if the propagation of the oscillation is unidirectional from CA3 to CA1, applying gabazine locally to CA1 should not affect the oscillation in CA3. Indeed, local drug application immediately abolished the oscillation in CA1 (the PSD peak amplitude changed to $17 \pm 5 \%$ of the control, $p<0.001, n=4$, paired $t$ test) but left the oscillation in CA3 intact ( $95 \pm 5 \%$ of control, $n=23$, paired $t$ test). Cessation of gabazine pressure ejection allowed for the complete recovery of the oscillation within a few minutes in the CA1 region $(106 \pm 18 \%$ compared with control, $p=0.75$, $n=4$, paired $t$ test) (Fig. $11 A-C$ ). These data show the effectiveness of local gabazine application to eliminate the main local current source of gamma oscillation under our recording conditions, and confirm that oscillations monitored in CA3 can be used as a reference signal for detecting changes in spiking of CA1 neurons upon blocking inhibition within CA1.

In the next set of experiments, we recorded the firing activity of CA1 PCs and PV + INs in a loose-patch mode, while the field oscillation was continuously monitored in CA3. Both CA1 PCs and $\mathrm{PV}+\mathrm{INs}$ showed weaker phase-coupling to the oscillation recorded in CA3 $\left(r_{\mathrm{AP}}=0.07 \pm 0.02, n=14\right.$ and $0.35 \pm 0.07, n=$ 9 , respectively, including nonphase-coupled cells) compared with those phase-coupling values that were obtained in relation to CA1 oscillation (CA1 PCs: $r_{\mathrm{AP}}=0.16 \pm 0.02, n=21, p<0.01$; PV + INs: $r_{\mathrm{AP}}=0.75 \pm 0.04, n=11, p<0.001$; two-sample $t$ test). From the $14 \mathrm{PCs}$ recorded in this part of the study, only four neurons showed phase-coupled firing $\left(p_{\mathrm{r}}<0.01\right)$, which fired close to the trough of the oscillation monitored in CA3 $\left(\Phi_{\mathrm{AP}}=\right.$ $-2.35 \pm 0.18 \mathrm{rad}$ ). After gabazine application, all these four CA1 PCs remained phase-coupled (in three of them $p_{\mathrm{r}}<0.01$, whereas in one of them $\left.p_{\mathrm{r}}=0.05\right)$; however, they all changed the phase of firing. Instead of firing at the trough, these CA1 PCs started to fire close to the peak of the field oscillation $\left(\Phi_{\mathrm{AP}}=0.07 \pm 0.36 \mathrm{rad}\right.$, $p<0.01, n=4$, paired-sample circular test; Fig. $11 D, G)$. Of the 10 remaining PCs that showed no phase-coupling under control conditions, half of them showed no changes in the firing pattern. The other half, however, started to fire phase-locked, but again close to the peak, instead of the trough of the oscillation $\left(\Phi_{\mathrm{AP}}=\right.$ $0.48 \pm 0.32, n=5)$. Interestingly, the firing frequency of CA1 PCs did not change after gabazine application $(6.25 \pm 0.62 \mathrm{~Hz}$ in control and $6.31 \pm 0.66 \mathrm{~Hz}$ in gabazine, $p=0.85, n=14$, paired $t$ test). These results suggest that in the lack of local inhibition, the discharge of CA1 PCs is more likely to be driven by CA3 excitatory input. However, under normal conditions, when inhibition is intact in the slices, synaptic inhibition originated from local INs 

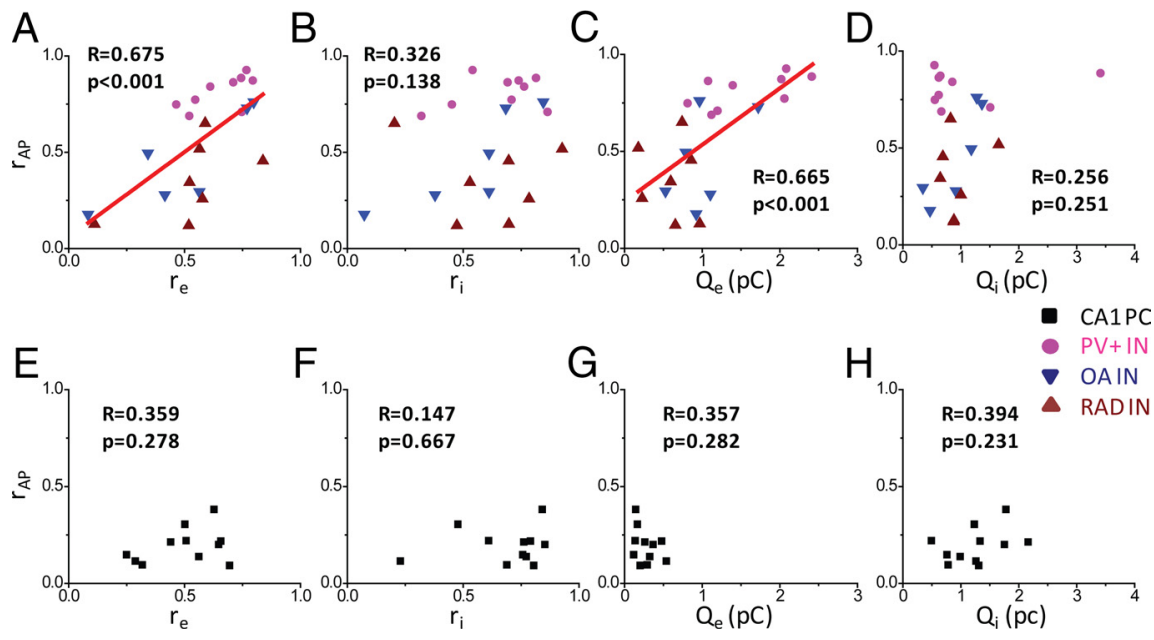

Figure 9. Correlations between the strength of action potential phase-coupling $\left(r_{\mathrm{AP}}\right)$ and the synaptic inputs of the CA1 cells. The phase-coupling strength of the action potentials plotted against the phase-coupling strength of excitatory $\left(r_{\mathrm{e}}\right)$ and inhibitory $\left(r_{i}\right)$ inputs and phasic excitatory $\left(Q_{\mathrm{e}}\right)$ and inhibitory $\left(Q_{j}\right)$ charge transfer for individual CA1 INs $(\boldsymbol{A}-\boldsymbol{D}$, respectively) and PCS (E-H, respectively). Significant correlations were found only between the properties of excitatory inputs (both $r_{\mathrm{e}}$ and $Q_{\mathrm{e}}$ ) and the $r_{\mathrm{AP}}$ of INs. Different cell types are marked with different symbols (Pearson's correlation).
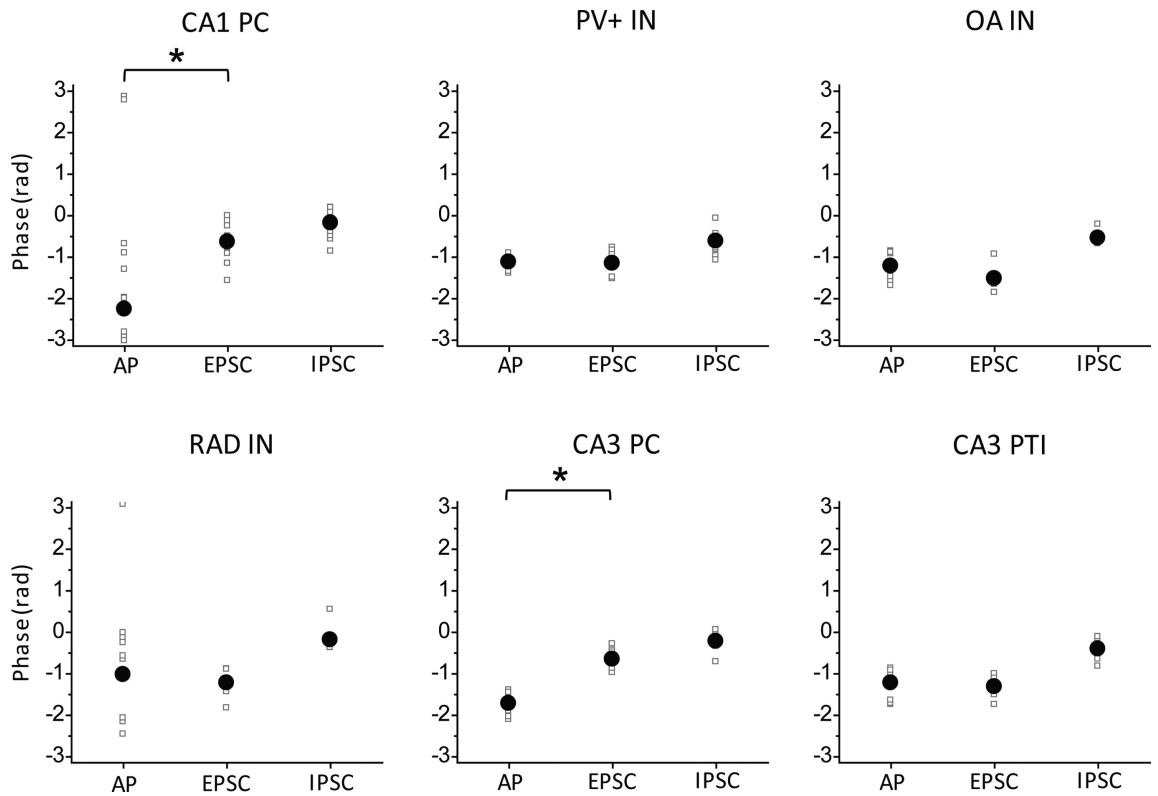

Figure 10. Phase of firing [action potential (AP)], peak excitation (EPSC), and peak inhibition (IPSC) in phase-coupled neurons. Note that all neuron types both in CA1 and CA3 received both EPSCs and IPSCs in comparable phases of the gamma cycles. The action potentials tended to appear just after the peak excitation in the majority of INs; however, spiking occurred much earlier than the peak excitation in the case of CA1 PCS, CA3 PCS, and some RAD INs. Means are indicated with black dots. Asterisks indicate the significant differences.

dominates over the excitatory drive, determining the firing phase of phase-locked CA1 PCs.

The firing of all CA1 PV+ INs was phase-coupled to the ascending phase of the field oscillation detected in CA3 $\left(\Phi_{\mathrm{AP}}=\right.$ $-0.98 \pm 0.18, n=9$ ). After local application of gabazine into CA1, CA1 PV+ INs showed a small shift in the phase of their firing toward the peak of the oscillatory cycle $\left(\Phi_{\mathrm{AP}}=-0.64 \pm\right.$ $0.21, p<0.001, n=9$, paired-sample circular test), but they were still firing at the ascending phase without changing their firing rate $(14.30 \pm 3.42 \mathrm{~Hz}$ in control and $15.73 \pm 3.57 \mathrm{~Hz}$ in gabazine, $p=0.31, n=9$ paired $t$ test), or their phase-coupling strength $\left(r_{\mathrm{AP}}=0.34 \pm 0.07\right.$ in control and $0.34 \pm 0.04$ in gabazine, $p=$
$0.91, n=9$ paired $t$ test) (Fig. $11 E, H)$. In five of the nine CA1 PV+ INs, we recorded EPSCs both in control conditions and during gabazine application. Blocking the local inhibition in CA1 did not change the excitatory inputs in PV+ INs. In these interneurons, both the phase and the strength of the phase-coupling of the peak excitation, as well as the phasic charge transfer, remained unchanged $\left(\Phi_{\mathrm{e}}=\right.$ $-1.30 \pm 0.19 \mathrm{rad}$ in control and $\Phi_{\mathrm{e}}=$ $-1.18 \pm 0.30 \mathrm{rad}$ in gabazine, $p=0.79$, paired-sample circular test; $r_{\mathrm{e}}=0.60 \pm$ 0.06 in control and $r_{\mathrm{e}}=0.47 \pm 0.06$ in gabazine, $p=0.13 ; \mathrm{Qe}=0.71 \pm 0.19 \mathrm{pC}$ in control and $Q \mathrm{e}=0.660 \pm 0.13 \mathrm{pC}$ in gabazine, $p=0.55, n=5$, paired $t$ tests) (Fig. 11F,I). Nevertheless, application of gabazine effectively decreased the inhibitory charge transfer in PV+ INs to $17 \%$ of control values $(n=4, p<0.05$, paired $t$ test), showing that puffing gabazine locally into CA1 effectively eliminates the inhibitory currents.

These results suggest that during CChinduced oscillations, the Schaffer collaterals provide the major contribution in controlling CA1 IN spike timing, with a lesser contribution provided by inhibitory input. The surprising findings that in the absence of inhibition CA1 PC firing became more akin to that of CA1 INs, as well as the fact that excitatory input to CA1 INs was unchanged, strongly support the hypothesis that the synaptic excitation driving the discharge of CA1 INs in the in vitro network is predominantly of CA3 origin (Fig. 12).

\section{Discussion}

We found the following: (1) The majority of CA1 INs were strongly coupled to the local oscillation in contrast to CA1 PCs, which showed weak phase-coupling. (2) All neurons in both CA1 and CA3 received strongly phase-coupled excitatory drive on the ascending phase of the oscillation cycle. (3) The dominant input to CA1 INs was excitatory and originated from the CA3 PCs. Firing properties correlated with the properties of this excitatory drive, and excitation preceded firing. (4) There was no correlation between CA1 PC firing characteristics and excitatory input properties. (5) Inhibitory input also contributed to controlling the phase of the firing of CA1 neurons.

Gamma oscillations generated in CA3 can propagate to CA1 (Fisahn et al., 1998); however, the mechanisms by which CA3 output recruits elements in the CA1 network resulting in a local oscillation have not been known. Our data suggest that CA1 INs receive a common excitatory input, supporting the conclusion that Schaffer collaterals provide a source of strong phasic glutamatergic drive to these cells. We report a $0.7 \mathrm{~ms}$ time lag between CA1 and $\mathrm{CA} 3$ oscillations, which is in agreement with the difference in 
A control

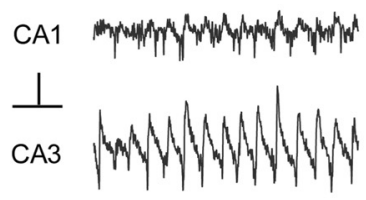

gabazine in CA1

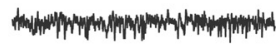

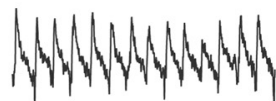

washout

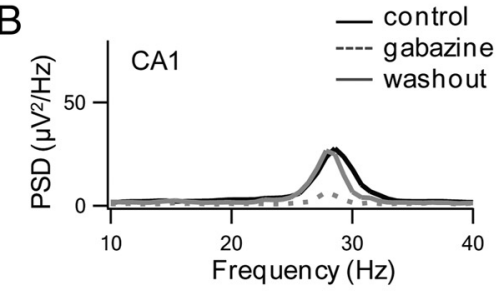

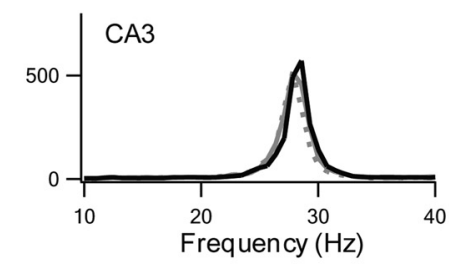

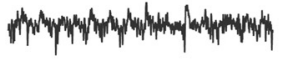
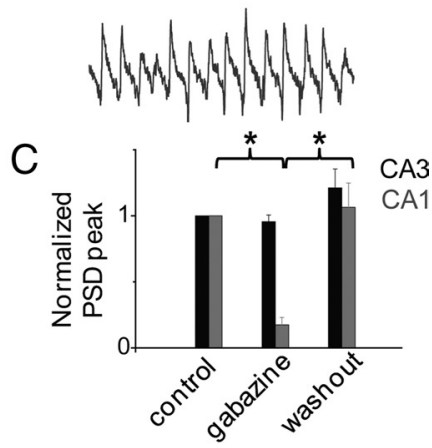

control

gabazine in CA1

D CA1 PC spikes
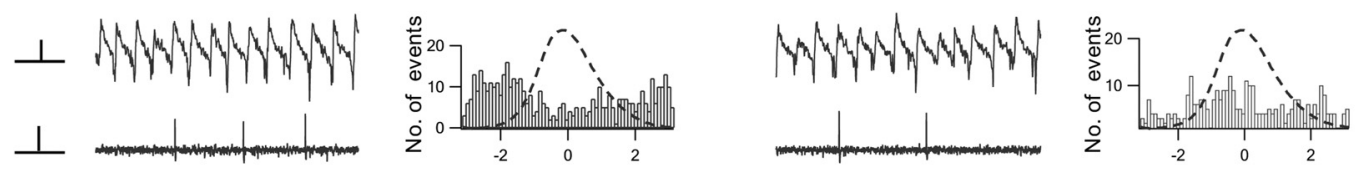

E CA1 PV+ IN spikes
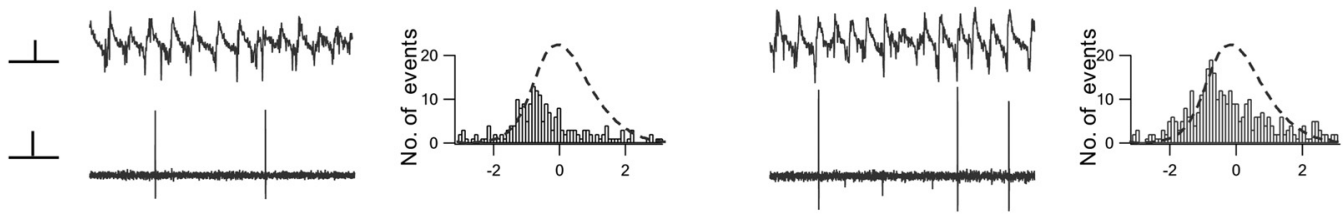

F

CA1 PV+ IN EPSC

$\perp$ MMAMWHAWHAW

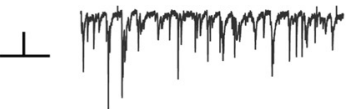
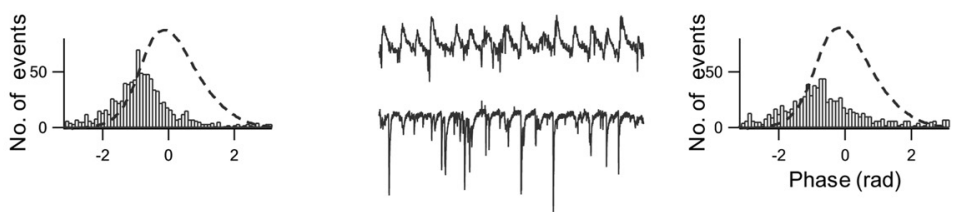

G

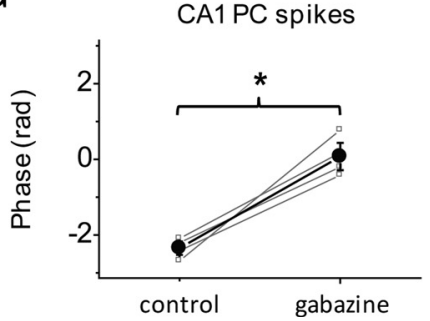

$\mathrm{H}$

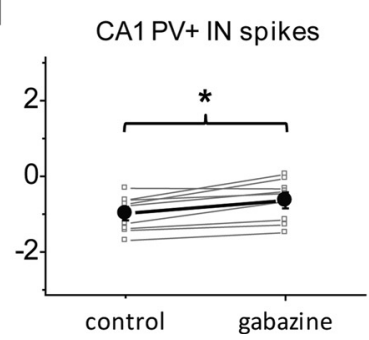

I

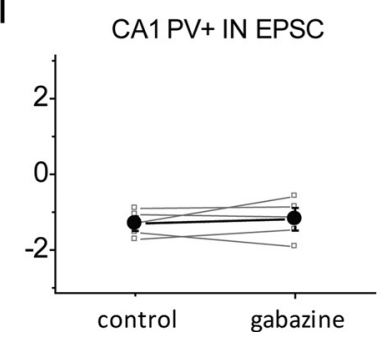

Figure 11. Local CA1 pressure ejection of GABA receptor antagonist diminishes oscillation power in this subfield and alters the firing of CA1 PCs and PV+ INs without changing the properties of phasic excitatory inputs in these interneurons. $A$, Raw traces of field potential oscillations recorded simultaneously from the stratum pyramidale in CA1 (top) and CA3 (bottom) in the control condition (10 $\mu \mathrm{m} C \mathrm{Ch}$, left), during local gabazine application onto CA1 (50 $\mu \mathrm{m}$, middle), and after gabazine washout (right). $\boldsymbol{B}$, Power spectral density functions of the traces in $\boldsymbol{A}$ under the control condition (black lines), during gabazine-puff (dotted lines), and after washout (gray lines). C, Comparison of the power of oscillations under the different conditions in CA3 (black) and CA1 (gray). Data were normalized to the control values. Local gabazine-puffin CA1 significantly reduced the power of the oscillation in $C A 1(n=4)$, but notin $C A 3(n=23)$. D, Extracellular recordings of field potentials in the stratum pyramidale of $C A 3$ (top trace) with simultaneously recorded spikes from a CA1 PC (loose-patch recordings, bottom trace). Spike-phase histograms of the same neuron under the control condition (left) and during local gabazine-puffi in CA1 (right). E, Same plots as in D but for a CA1 PV + INs. F, Simultaneous recordings of field potential oscillations in the stratum pyramidale of CA3 (top trace) and EPSCS (bottom trace) detected in the same CA1 PV + INs as in $\boldsymbol{E}$ under the control condition (left) and during local gabazine-puff in CA1 (right). EPSCs were recorded in whole-cell voltage-clamp mode at a holding potential of - $80 \mathrm{mV}$. A phase histogram of EPSCs obtained from recordings under the control condition (left) and during local gabazine-puffin CA1 (right) is shown. $\boldsymbol{G}, \boldsymbol{H}$, Preferred phases of firing of phase-coupled CA1 PCS $(\boldsymbol{G})$ and CA1 PV + INs $(\boldsymbol{H})$ under control conditions and during local gabazine-puff in CA1. Note that CA1 PCs changed the phase of firing from the trough to the peak of the oscillation. $I$, The preferred phases of the peak excitation received by PV + INs in CA1 during oscillation under the control condition and during local gabazine-puff to the CA1 region. Note that application of gabazine did not influence the phase of excitation. The dashed lines in $D-G$ indicate the mean gamma cycle. Calibration: vertical, $0.1 \mathrm{mV}$ for extracellular field-recordings, $0.2 \mathrm{mV}$ for loose-patch recordings of spiking activity, and $50 \mathrm{pA} \mathrm{for} \mathrm{voltage-clamp} \mathrm{recordings} \mathrm{of} \mathrm{EPSCS;} \mathrm{horizontal,} 0.1 \mathrm{~s}$. Asterisks indicate significant changes according to paired sample $t$ tests $(\boldsymbol{C})$ and circular tests $(\mathbf{G}, \boldsymbol{H})$. 


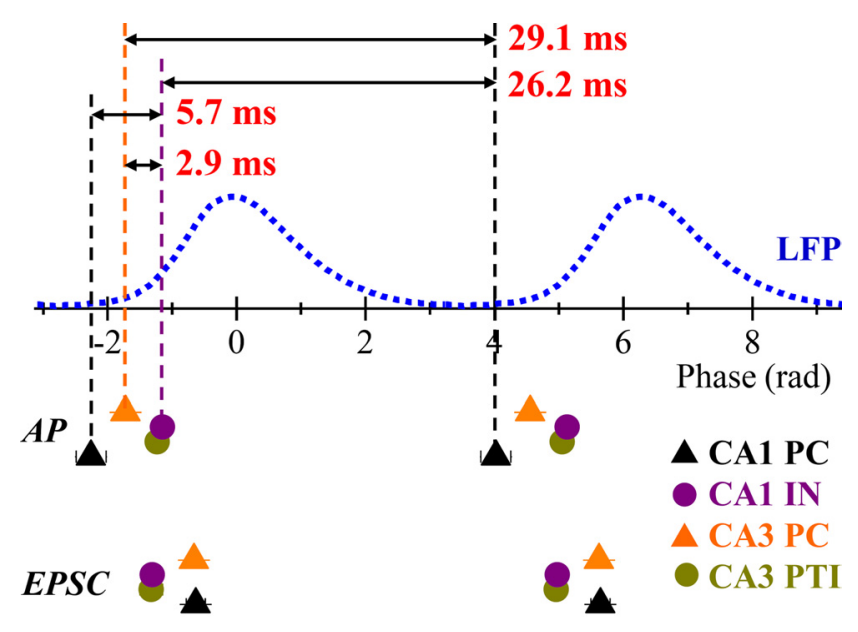

IPSC

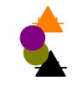

Figure 12. The time differences between the action potentials and the synaptic events recorded in the different cell types support a model whereby gamma oscillations propagate from the $C A 3$ area to the $C A 1$ region of the hippocampus via feedforward inhibition. According to our results, the average time difference between firing of CA3 PCs and INs in CA1 and CA3 is $2.9 \mathrm{~ms}$, whereas the time difference between the firing of CA1 PCs and INs is $5.7 \mathrm{~ms}$, on average. Relative firing times of CA3 and CA1 PCs (on average, $29.1 \mathrm{~ms}$ ) are inconsistent with a direct feedforward excitation generating CA1 PC action potentials. These results suggest that the discharge of CA1 INs, like CA3 INs, is driven directly by their excitatory inputs from CA3 PCs. Synaptic inhibition controls the firing time of CA1 PCs, resulting in an average time delay of 26.2 ms between CA1 INs and CA1 PC firing. Symbols indicate the mean phases ( \pm SEM) of the action potentials, peak excitation (EPSC), and peak inhibition (IPSC) in the different cell groups. The dotted blue line shows two cycles of the averaged LFP oscillation in CA1.

time of an action potential propagating along the CA3 recurrent collaterals versus Schaffer collaterals $(0.5-1.5 \mathrm{~mm})$ with a conduction velocity of $0.5 \mathrm{~mm} / \mathrm{ms}$ (Meeks and Mennerick, 2007). This is also in the order of the time difference between EPSCs in CA3 PTIs and CA1 PV+ INs of $0.82 \mathrm{~ms}$ (based on a cycle period of $31 \mathrm{~Hz}$ ).

The results of our experiments, in which inhibition was blocked locally in CA1, showed that the CA3 excitatory output plays a major role in driving the firing of CA1 INs, although inhibition is also involved in controlling the precise spike timing of these cells. In contrast, CA3 excitatory input is not sufficient to control the firing of CA1 PCs when inhibition is intact. The broad phase tuning of CA1 PCs suggests that recurrent feedback excitation does not provide a major contribution to the phasic excitatory input of local INs, despite abundant recurrent connections between CA1 PCs and INs (Takács et al., 2012). Whereas gamma oscillations in CA3 are generated by reciprocal recurrent feedback mechanisms (Oren et al., 2006), our data point to a model in which local oscillations in CA1 are generated by rhythmic recruitment of feedforward inhibition and demonstrate the importance of inhibitory recruitment in coupling oscillatory function interregionally (Akam et al., 2012) (Fig. 12).

When comparing the synaptic inputs of the different cell types during oscillations, we found a striking difference in the magnitude of synaptic excitation recorded in CA1 PCs and INs, in line with previous results obtained in CA3 (Oren et al., 2006). Since PCs receive Schaffer collateral input mainly on their dendritic spines (Gulyás et al., 1999; Megías et al., 2001), it is probable that space-clamp limitations could influence our measurements. However, such a consideration does not affect the conclusion that the weak somatic excitatory currents in PCs are not sufficient to precisely control firing in these cells during CCh-induced gamma oscillations. In addition, systematic differences have been reported in the kinetic parameters of the excitatory postsynaptic currents between PCs and INs, showing that EPSCs in PCs have slower rise and decay kinetics than in INs (Geiger et al., 1995; Pouille and Scanziani, 2001). Such factors are also likely to contribute to the differences in synaptic properties between PCs and INs observed here.

Surprisingly, even OA INs in CA1 appeared to receive their main excitatory input from CA3 PCs in this gamma oscillation model, as both their firing phase and the phase of their excitatory input did not significantly differ from those recorded in PV+ INs or RAD INs. These results seem to contradict previous data implying that OA INs are feedback inhibitory cells, since they receive $60-70 \%$ of their glutamatergic inputs from their main target cells, i.e., CA1 pyramidal cells (Blasco-Ibáñez and Freund, 1995), which neurons excite them effectively (Maccaferri and McBain, 1995). A recent study, however, uncovered that both CA3 and CA1 PCs synapse onto OA INs, and these synapses differ in their receptor expression pattern and also in their plasticity properties (Croce et al., 2010). These cell-type- and afferentspecific rules of synaptic transmission and plasticity point to differential recruitment of OA INs in network activity under distinct conditions. Such differential recruitment has important implications for network output (Lovett-Barron et al., 2012).

Although cholecystokinin-expressing interneurons form a significant population of inhibitory cells in the hippocampus (Freund and Buzsáki, 1996), we would not expect that these GABAergic cells contribute significantly to oscillogenesis. Previous studies showed that endocannabinoids released from PCs after CCh treatment block GABA release from the axon terminals of cholecystokinin-expressing cells via activation of presynaptically located $\mathrm{CB}_{1}$ cannabinoid receptors (Fukudome et al., 2004; Neu et al., 2007; Gulyás et al., 2010). Thus, the muted output of these GABAergic interneurons in the presence of CCh makes it unlikely that the activity of cholecystokinin-containing cells is directly involved in cholinergically induced oscillations.

By comparing the inhibitory inputs of the neurons, we found that the absolute inhibitory charge was much larger in CA3 PCs than in any other cell types. This observation is in agreement with the recurrent model of gamma oscillogenesis in CA3 (Oren et al., 2006). Whereas the amount of phasic inhibitory charge was smaller in CA1 PCs than in CA3 PCs, no significant difference could be found in the ratio of phasic excitatory to inhibitory charge between the two PC populations. INs are likely to receive synaptic inhibition from numerous subpopulations of GABAergic cells, yet the inhibitory inputs were rather homogenous among them in both precision and timing. Although the mean of peak inhibition was somewhat later in OA INs and PV + INs in CA1 than in the other cell types, these differences could be explained by the diversity of IPSC kinetics of the various types of hippocampal INs (Hájos and Mody, 1997; Cossart et al., 2006).

Whereas the discharge of CA1 INs correlated with their phasic excitatory, but not inhibitory, drive, neither the excitatory nor the inhibitory synaptic input properties correlated with the firing characteristics of CA1 PCs. Although the dominant input recorded in CA1 and CA3 PCs during ongoing oscillation was inhibitory, their firing properties differed in terms of modulation depth and phase-coupling. One factor that could underlie the different spiking behavior may derive from the distinct effect of cholinergic receptor activation on the excitability of CA1 and CA3 PCs (Dasari and Gulledge, 2011). In addition, the difference in the absolute charge of phasic inhibition received by these neu- 
ron types could also contribute to their distinct discharge features. What might be the advantage of the weakly phase-coupled firing of CA1 PCs during CA3-driven gamma oscillation? The weak coupling might be important for making these cells capable of responding readily to excitatory input originating from the entorhinal cortex (Moser et al., 2008) and thus can promote the creation of temporal neuronal ensembles during attentive network states (Harris and Thiele, 2011).

The CCh-induced gamma oscillations in CA1 shared many features of hippocampal gamma oscillations recorded in vivo (Csicsvári et al., 2003). First, the firing of both PCs and INs tends to be phase-locked to gamma oscillations, and the proportion of gamma-modulated cells is higher for INs in CA1 compared with CA1 PCs both in the behaving animal and in our in vitro oscillation model. Second, the sequence of the discharge of the different cell types during a gamma cycle observed in vivo is similar to our observations. The spiking probability of CA1 PCs in the gamma cycles reaches its maximum earlier than INs. Whereas both CA1 and CA3 INs discharge after CA3 PCs with time lags accounting for monosynaptic delay, the time lag between the discharge of CA1 PCs and CA1 INs is too long to be taken as a monosynaptic excitation. Finally, PV+ INs show strong phase-coupling to the ongoing gamma oscillation both in vivo and during in vitro experiments (Bibbig et al., 2007; Tukker et al., 2007). These observations propose that $\mathrm{CCh}$-induced network oscillations provide an appropriate model for in vivo hippocampal gamma oscillations that are generated intrinsically in the CA3 region and propagate to CA1 (Bragin et al., 1995; Csicsvári et al., 2003; Isomura et al., 2006; Colgin et al., 2009).

In conclusion, our findings support a hypothesis that the intrahippocampal spread of gamma oscillation from CA3 to CA1 is mediated by feedforward excitation of CA1 INs (Fig. 12). The synchronized inhibitory postsynaptic currents originating from the rhythmic discharge of CA1 INs could play a major role in the generation of local field potential oscillation, as in the case of CA3 (Oren et al., 2010). Our results for the first time elucidate the synaptic mechanisms underlying in the propagation of oscillations between hippocampal CA3 and CA1. Since gamma band synchronization has been proposed to be involved in numerous brain functions (Fries, 2009), an understanding of the propagation of oscillations will be invaluable in revealing the functional role of these oscillations.

\section{References}

Akam T, Oren I, Mantoan L, Ferenczi E, Kullmann DM (2012) Oscillatory dynamics in the hippocampus support dentate gyrus-CA3 coupling. Nat Neurosci 15:763-768. CrossRef Medline

Bibbig A, Middleton S, Racca C, Gillies MJ, Garner H, Lebeau FE, Davies CH, Whittington MA (2007) Beta rhythms $(15-20 \mathrm{~Hz})$ generated by nonreciprocal communication in hippocampus. J Neurophysiol 97:2812-2823. CrossRef Medline

Blasco-Ibáñez JM, Freund TF (1995) Synaptic input of horizontal interneurons in stratum oriens of the hippocampal CA1 subfield:structural basis of feed-back activation. Eur J Neurosci 7:2170-2180. CrossRef Medline

Bragin A, Jandó G, Nádasdy Z, Hetke J, Wise K, Buzsáki G (1995) Gamma $(40-100 \mathrm{~Hz})$ oscillation in the hippocampus of the behaving rat. J Neurosci 15:47-60. Medline

Colgin LL, Denninger T, Fyhn M, Hafting T, Bonnevie T, Jensen O, Moser MB, Moser EI (2009) Frequency of gamma oscillations routes flow of information in the hippocampus. Nature 462:353-357. CrossRef Medline

Cossart R, Petanjek Z, Dumitriu D, Hirsch JC, Ben-Ari Y, Esclapez M, Bernard C (2006) Interneurons targeting similar layers receive synaptic inputs with similar kinetics. Hippocampus 16:408-420. CrossRef Medline

Croce A, Pelletier JG, Tartas M, Lacaille JC (2010) Afferent-specific properties of interneuron synapses underlie selective long-term regu- lation of feedback inhibitory circuits in CA1 hippocampus. J Physiol 588:2091-2107. CrossRef Medline

Csicsvári J, Jamieson B, Wise KD, Buzsáki G (2003) Mechanisms of gamma oscillations in the hippocampus of the behaving rat. Neuron 37:311-322. CrossRef Medline

Dasari S, Gulledge AT (2011) M1 and M4 receptors modulate hippocampal pyramidal neurons. J Neurophysiol 105:779-792. CrossRef Medline

Fisahn A, Pike FG, Buhl EH, Paulsen O (1998) Cholinergic induction of network oscillations at $40 \mathrm{~Hz}$ in the hippocampus in vitro. Nature 394: 186-189. CrossRef Medline

Fisher NI (1993) Statistical analysis of circular data. Cambridge, UK: Cambridge UP.

Freund TF, Buzsáki G (1996) Interneurons of the hippocampus. Hippocampus 6:345-470. Medline

Fries P (2009) Neuronal gamma-band synchronization as a fundamental process in cortical computation. Annu Rev Neurosci 32:209-224. CrossRef Medline

Fukudome Y, Ohno-Shosaku T, Matsui M, Omori Y, Fukaya M, Tsubokawa H, Taketo MM, Watanabe M, Manabe T, Kano M (2004) Two distinct classes of muscarinic action on hippocampal inhibitory synapses: M2mediated direct suppression and M1/M3-mediated indirect suppression through endocannabinoid signalling. Eur J Neurosci 19:2682-2692. CrossRef Medline

Geiger JR, Melcher T, Koh DS, Sakmann B, Seeburg PH, Jonas P, Monyer H (1995) Relative abundance of subunit mRNAs determines gating and $\mathrm{Ca} 2+$ permeability of AMPA receptors in principal neurons and interneurons in rat CNS. Neuron 15:193-204. CrossRef Medline

Gulyás AI, Megías M, Emri Z, Freund TF (1999) Total number and ratio of excitatory and inhibitory synapses converging onto single interneurons of different types in the CA1 area of the rat hippocampus. J Neurosci 19: 10082-10097. Medline

Gulyás AI, Szabó GG, Ulbert I, Holderith N, Monyer H, Erdélyi F, Szabó G, Freund TF, Hájos N (2010) Parvalbumin-containing fast-spiking basket cells generate the field potential oscillations induced by cholinergic receptor activation in the hippocampus. J Neurosci 30:15134-15145. CrossRef Medline

Hájos N, Mody I (1997) Synaptic communication among hippocampal interneurons: properties of spontaneous IPSCs in morphologically identified cells. J Neurosci 17:8427-8442. Medline

Hájos N, Mody I (2009) Establishing a physiological environment for visualized in vitro brain slice recordings by increasing oxygen supply and modifying aCSF content. J Neurosci Methods 183:107-113. CrossRef Medline

Hájos N, Paulsen O (2009) Network mechanisms of gamma oscillations in the CA3 region of the hippocampus. Neural Netw 22:1113-1119. CrossRef Medline

Hájos N, Pálhalmi J, Mann EO, Németh B, Paulsen O, Freund TF (2004) Spike timing of distinct types of GABAergic interneuron during hippocampal gamma oscillations in vitro. J Neurosci 24:9127-9137. CrossRef Medline

Hájos N, Ellender TJ, Zemankovics R, Mann EO, Exley R, Cragg SJ, Freund TF, Paulsen O (2009) Maintaining network activity in submerged hippocampal slices: importance of oxygen supply. Eur J Neurosci 29:319-327. CrossRef Medline

Harris KD, Thiele A (2011) Cortical state and attention. Nat Rev Neurosci 12:509-523. CrossRef Medline

Isomura Y, Sirota A, Ozen S, Montgomery S, Mizuseki K, Henze DA, Buzsáki G (2006) Integration and segregation of activity in entorhinal-hippocampal subregions by neocortical slow oscillations. Neuron 52:871-882. CrossRef Medline

Le Van Quyen M, Foucher J, Lachaux J, Rodriguez E, Lutz A, Martinerie J, Varela FJ (2001) Comparison of Hilbert transform and wavelet methods for the analysis of neuronal synchrony. J Neurosci Methods 111:83-98. CrossRef Medline

Li XG, Somogyi P, Ylinen A, Buzsáki G (1994) The hippocampal ca3 network - an in vivo intracellular labeling study. J Comp Neurol 339: 181-208. CrossRef Medline

Lovett-Barron M, Túri GF, Kaifosh P, Lee PH, Bolze F, Sun XH, Nicoud JF, Zemelman BV, Sternson SM, Losonczy A (2012) Regulation of neuronal input transformations by tunable dendritic inhibition. Nat Neurosci 15: 423-430. CrossRef Medline

Maccaferri G, McBain CJ (1995) Passive propagation of LTD to stratum 
oriens-alveus inhibitory neurons modulates the temporoammonic input to the hippocampal CA1 region. Neuron 15:137-145. CrossRef Medline

Mann EO, Suckling JM, Hájos N, Greenfield SA, Paulsen O (2005) Perisomatic feedback inhibition underlies cholinergically induced fast network oscillations in the rat hippocampus in vitro. Neuron 45:105-117. CrossRef Medline

McBain CJ, DiChiara TJ, Kauer JA (1994) Activation of metabotropic glutamate receptors differentially affects two classes of hippocampal interneurons and potentiates excitatory synaptic transmission. J Neurosci 14:4433-4445. Medline

Meeks JP, Mennerick S (2007) Action potential initiation and propagation in CA3 pyramidal axons. J Neurophysiol 97:3460-3472. CrossRef Medline

Megías M, Emri Z, Freund TF, Gulyás AI (2001) Total number and distribution of inhibitory and excitatory synapses on hippocampal CA1 pyramidal cells. Neuroscience 102:527-540. CrossRef Medline

Meyer AH, Katona I, Blatow M, Rozov A, Monyer H (2002) In vivo labeling of parvalbumin-positive interneurons and analysis of electrical coupling in identified neurons. J Neurosci 22:7055-7064. Medline

Minlebaev M, Colonnese M, Tsintsadze T, Sirota A, Khazipov R (2011) Early gamma oscillations synchronize developing thalamus and cortex. Science 334:226-229. CrossRef Medline

Montgomery SM, Buzsáki G (2007) Gamma oscillations dynamically couple hippocampal CA3 and CA1 regions during memory task performance. Proc Natl Acad Sci U S A 104:14495-14500. CrossRef Medline

Moser EI, Kropff E, Moser MB (2008) Place cells, grid cells, and the brain's spatial representation system. Annu Rev Neurosci 31:69-89. CrossRef Medline

Neu A, Földy C, Soltész I (2007) Postsynaptic origin of CB1-dependent tonic inhibition of GABA release at cholecystokinin-positive basket cell to pyramidal cell synapses in the CA1 region of the rat hippocampus. J Physiol 578:233-247. Medline
Oren I, Mann EO, Paulsen O, Hájos N (2006) Synaptic currents in anatomically identified CA3 neurons during hippocampal gamma oscillations in vitro. J Neurosci 26:9923-9934. CrossRef Medline

Oren I, Hájos N, Paulsen O (2010) Identification of the current generator underlying cholinergically induced gamma frequency field potential oscillations in the hippocampal CA3 region. J Physiol 588:785-797. CrossRef Medline

Paulsen O, Moser EI (1998) A model of hippocampal memory encoding and retrieval: GABAergic control of synaptic plasticity. Trends Neurosci 21:273-278. CrossRef Medline

Pouille F, Scanziani M (2001) Enforcement of temporal fidelity in pyramidal cells by somatic feed-forward inhibition. Science 293:1159-1163. CrossRef Medline

Singer W (1993) Synchronization of cortical activity and its putative role in information processing and learning. Annu Rev Physiol 55:349-374. CrossRef Medline

Somogyi P, Klausberger T (2005) Defined types of cortical interneurone structure space and spike timing in the hippocampus. J Physiol 562: 9-26. CrossRef Medline

Takács VT, Klausberger T, Somogyi P, Freund TF, Gulyás AI (2012) Extrinsic and local glutamatergic inputs of the rat hippocampal CA1 area differentially innervate pyramidal cells and interneurons. Hippocampus 22 : 1379-1391. CrossRef Medline

Tukker JJ, Fuentealba P, Hartwich K, Somogyi P, Klausberger T (2007) Cell type-specific tuning of hippocampal interneuron firing during gamma oscillations in vivo. J Neurosci 27:8184-8189. CrossRef Medline

Zar JH (1999) Biostatistical analysis. Upper Saddle River, NJ: Prentice Hall.

Zemankovics R, Káli S, Paulsen O, Freund TF, Hájos N (2010) Differences in subthreshold resonance of hippocampal pyramidal cells and interneurons: the role of h-current and passive membrane characteristics. J Physiol 588:2109-2132. CrossRef Medline 